\title{
Reconfiguring Smart Structures using Approximate Heteroclinic Connections
}

\author{
Jiaying Zhang ${ }^{1,2}$ and Colin R McInnes ${ }^{2}$ \\ ${ }^{1}$ Department of Mechanical and Aerospace Engineering, University of Strathclyde, Glasgow, G1 1XJ, \\ United Kingdom \\ ${ }^{2}$ School of Engineering, University of Glasgow, Glasgow, G12 8QQ, United Kingdom \\ E-mail: jiaying.zhang@strath.ac.uk
}

\begin{abstract}
A new method is investigated to reconfigure smart structures by the technique of polynomial series, used to approximate a true heteroclinic connection between unstable equilibria in a smart structure model. We explore the use of polynomials of varying order to firstly approximate the heteroclinic connection between two equalenergy unstable equilibrium points, and then develop an inverse method to control the dynamics of the system to track the reference polynomial trajectory. It is found that high order polynomials can provide a good approximation to heteroclinic connections and provide an efficient means of generating such trajectories. The method is used firstly in a simple smart structure model to illustrate the method and is then extended to a more complex model where the numerical generation of true heteroclinic connections is difficult. It is envisaged that being computationally efficient, the method could form the basis for real-time reconfiguration of smart structures using heteroclinic connections between equal-energy, unstable configurations.
\end{abstract}




\section{Introduction}

Significant research work currently focuses on new materials which have properties that can be changed in a controlled fashion by external stimuli, such as stress, temperature, electric or magnetic fields, for example shape memory materials (SMM) [1]. Materials can therefore be designed and manufactured with desirable mechanical properties which can then be used to develop smart structures [2]. Applications include vibration control and active shape control of flexible structures such as plates and trusses [3], with significant experimental results demonstrating that flexible structural vibrations can be effectively reduced [4,5]. Other applications involve the use of smart materials in unstable systems to actively monitor and suppress instability in a controlled manner [6]. Smart structures can also be used for the detection of cracks and corrosion monitoring [7], while embedded sensors and actuators can be used to actively control bucking in a loaded beam [8]. Smart materials are also used in micromechanics for energy harvesting, which converts energy in one form to another, such as from mechanical movement into electric energy[9].

The active control of unstable smart structures has been investigated by Hogg and Huberman [10] using an agent-based approach to suppress instability. They considered how to improve control of a given shape in a smart structure and investigated the possibility of dynamically transitioning between two configurations of the structure, one of which is stable and the other unstable. An elastic continuous beam model with simply supported boundary conditions was also investigated by Camescasse, Fernandes and Pouget $[11,12]$ who used nonlinear theory to investigate the transition between two stable positions of a buckled beam and snap-through phenomenon. Yoon and Washington have shown that deformable structures can be reconfigured through an optimal method of shape control, and a mechanically deformable reflector antenna structure was simulated as an application $[13,14]$. Some applications of reconfiguring smart structures are also emerging to improve the aerodynamic and aeroelastic performance of aircraft[15]. Recently a new smart structure concept for self-folding origami has been presented, which can fold itself through embedded electronics into a desired shape [16]. A crawling robot that can fold itself was developed to demonstrate the application of this technique to the fabrication of reconfigurable machines [17].

In this paper, previous work by McInnes and Waters [18] is firstly summarised. We use dynamical system theory to investigate the characteristics of their double mass-spring problem as a simple model of a smart structure. We then identify a set of both stable and unstable equilibrium configurations in the model and consider reconfiguration of the smart structure between the equal-energy unstable states. It is assumed that active control can maintain the structure in an unstable state [19]. A reconfigurable smart structure is defined here as a mechanical system which has the ability to change its kinematic configuration between a finite set of stable or unstable equilibria. To achieve such a reconfiguration here we attempt to connect the unstable equilibria through heteroclinic connections in the phase space of the problem. Because unstable equilibria can be found which lie on the same energy surface in the 
phase space, if a heteroclinic connection between unstable, equal energy equilibria can be defined, trajectories exist between these configurations which in principle do not require the addition of or dissipation of energy. Previous work [18] illustrated that the use of such heteroclinic connections between unstable equilibria can in principle be energetically efficient compared to reconfiguring a structure between stable configurations, which requires the addition of and then dissipation of energy.

However, it can be difficult to obtain such heteroclinic connections numerically in complex dynamical systems, such as those with strong nonlinearity. Therefore, in this paper, a reconfiguration method which is based on a reference trajectory and an inverse control method which is applied to the simple double mass-spring model of the smart structure. The principal advantage of the inverse method for this problem is the flexibility for path shaping. For example, a sufficiently smooth set of functions can be used to generate a path to approximate the heteroclinic connection and satisfy a number of boundary conditions for the problem. Again, it is envisaged that being computationally efficient, the method can form the basis for real-time reconfiguration of smart structures using heteroclinic connections between equal-energy, unstable configurations.

The fundamental theory of inverse control is then discussed and applied to the reconfiguration of the simple model of a smart structure. It is demonstrated that families of $4^{\text {th }}$ order polynomials can provide suitable reference functions to generate a phase space trajectory which approximates the real heteroclinic connection while satisfying the boundary conditions of the problem.

An evaluation criteria is then defined by again using a simple spring model, which under quasi-static conditions provides a relationship between the control action and the spring deformation required for control, thus developing a measure of the energy required for control. This evaluation criteria is then applied to the smart structure reconfiguration problem in order to assess the relative energy cost of different reconfiguration methods. We then consider a higher order polynomial whose additional free parameters allow an optimisation algorithm to be used to minimise the control effort required for reconfiguration. Some numerical results are then presented to elaborate on the feasibility of this reconfiguration manoeuvre. Finally, we extend the method from the double spring-mass model to a 3 mass problem which provides a significant step change in complexity, both in terms of the number of equilibrium states and in the difficulty of finding a true heteroclinic connection. However, it is demonstrated that the polynomial method and inverse control can provide effective reconfiguration of the structure between equal-energy unstable equilibria.

\section{Smart structure model}

In order to investigate how to use a polynomial as an approximation of a heteroclinic connection to reconfigure a smart structure, a simple representative model of a structure is defined [20]. We firstly assume a beam modelled as a single lumped mass and two linear springs to simulate the structure's 
stiffness, with both ends clamped, as shown in Fig. 1. The parameters of the model are the mass $m$ of the single lumped mass and the spring stiffness and natural length $k$ and $l$, respectively. The distance between the two clamped ends is $2 d$ and the displacement of the mass in the vertical direction is defined by $x$. From Fig. 1 it can be seen that the dynamics of the model can therefore be described by

$$
\begin{gathered}
\dot{x}=v \\
m \dot{v}=-2 k x\left(1-\frac{l}{\sqrt{x^{2}+d^{2}}}\right)
\end{gathered}
$$

Equation (2) can be expanded by assuming that $x / d \ll 1$ to obtain

$$
m \dot{v}=-2 k\left(\frac{l}{d}-1\right) x-\frac{k l}{d^{3}} x^{3}+\cdots
$$

which can be written as

$$
\begin{gathered}
\dot{q}=p \\
\dot{p}=\mu q-q^{3}
\end{gathered}
$$

where the non-dimensional position variable $q=\sqrt{l / d^{3}} x$ and non-dimensional time $\tau=t / \sqrt{m / k}$ are defined. The free parameter $\mu=2(l / d-1)$ is used as a measure of the compressive load acting on the model. Later, we will assume that the natural length of the spring can be manipulated through the use of an appropriate material, thus allowing active control of the structure. A relationship can be therefore be found between variations of $\Delta \mu$ and variations of the real spring length $\Delta l$, where $\delta$ can be defined as the ratio of total deformation to the initial length of the spring such that

$$
\delta=\frac{\Delta l}{l}=\frac{\Delta \mu}{\mu+2}
$$

Then, consider that the system is conservative so the problem also can be understood such that $\dot{p}=-\partial V(q, \mu) / \partial q$ through the use of an effective potential $V(q, \mu)$, defined as

$$
V(q, \mu)=-\frac{1}{2} \mu q^{2}+\frac{1}{4} q^{4}
$$




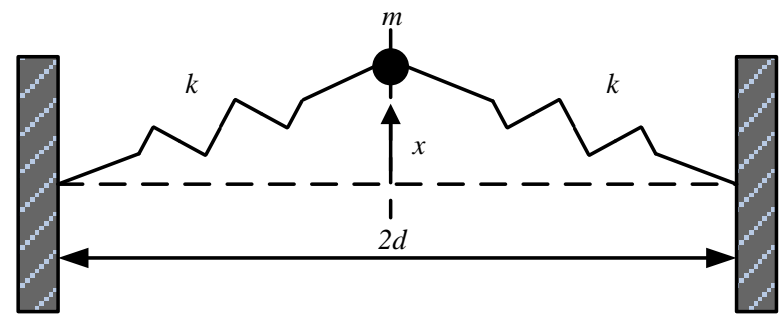

Figure 1. 1degree-of-freedom bucking beam model.

so that we can extend the 1 degree-of-freedom problem to a chain of $N$ masses. Considering that the system is still conservative we use the same functional form of nonlinearity as Eq. (7) above. The potential can then be generalised to arbitrary pairs of neighbouring masses $i-1$ and $i$ as

$$
V\left(q_{i-1}, q_{i}, \mu_{i}\right)=-\frac{1}{2} \mu_{i}\left(q_{i-1}-q_{i}\right)^{2}+\frac{1}{4}\left(q_{i-1}-q_{i}\right)^{4}
$$

In order to control the dynamics of the problem it will again be assumed that we can manipulate the coupling parameters $\mu_{i}$ to achieve active control of the structure. Since there is a linear relationship between $\mu_{i}$ and the natural length of the spring, we again assume that we can manipulate $\mu_{i}$ through changes to the natural length of the spring in the model. Now the behaviour of a chain of masses can be described by a Hamiltonian $H(\boldsymbol{q}, \boldsymbol{p}, \boldsymbol{\mu})=T(\boldsymbol{p})+V(\boldsymbol{q}, \boldsymbol{\mu})$ with the set $\boldsymbol{q}=\left\{q_{i}\right\}(i=1-\mathrm{N})$ and the corresponding set of momenta $\boldsymbol{p}=\left\{p_{i}\right\}(i=1-\mathrm{N})$ such that $(\boldsymbol{p}, \boldsymbol{q}) \in \mathbf{R}^{2 N}$, where $T(\boldsymbol{p})$ represents kinetic energy and $V(\boldsymbol{q}, \boldsymbol{\mu})$ represent potential energy where

$$
\begin{gathered}
T(\boldsymbol{p})=\frac{1}{2}\left\|\boldsymbol{p}^{2}\right\| \\
V(\boldsymbol{q}, \boldsymbol{\mu})=\sum_{i=1}^{N+1}-\frac{1}{2} \mu_{i}\left(q_{i-1}-q_{i}\right)^{2}+\frac{1}{4}\left(q_{i-1}-q_{i}\right)^{4}
\end{gathered}
$$

with boundary conditions $q_{0}=0$ and $q_{N}=0$, so that the chain is pinned at both ends.

In order to explore the possibility of reconfiguring a smart structure using the approximate polynomial method, a simple two masses chain with three linear springs will firstly be considered with the springs clamped at both ends, as shown in Fig. 2. The model assumes that the masses are constrained to move only in the vertical direction. 


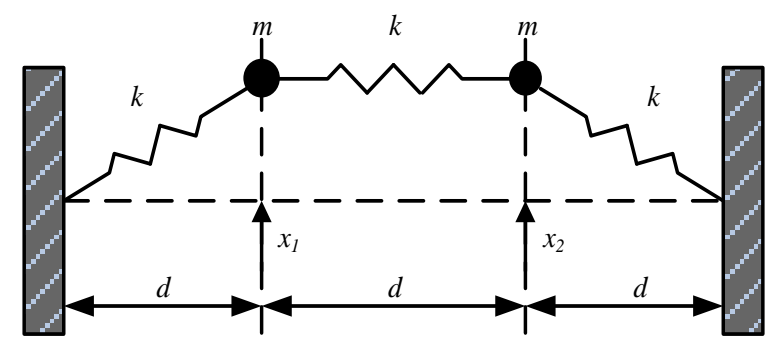

Figure 2. 2 degree-of-freedom bucking beam model.

The Hamiltonian for this two masses model can then be defined from the kinetic energy and potential energy through the Eqs. (9) and (10) as

$$
\begin{gathered}
T(\boldsymbol{p})=\frac{1}{2}\left|\boldsymbol{p}_{\mathbf{1}}{ }^{2}\right|+\left|\boldsymbol{p}_{\mathbf{2}}{ }^{2}\right| \\
V(\boldsymbol{q}, \boldsymbol{\mu})=-\frac{1}{2} \mu_{1} q_{1}{ }^{2}-\frac{1}{2} \mu_{2}\left(q_{1}-q_{2}\right)^{2}-\frac{1}{2} \mu_{3}{q_{2}}^{2}+\frac{1}{4} q_{1}{ }^{4}+\frac{1}{4}\left(q_{1}-q_{2}\right)^{4}+\frac{1}{4} q_{2}{ }^{4}
\end{gathered}
$$

Now we can fully define the problem by a dynamical system of the form

$$
\begin{gathered}
\dot{q}_{1}=p_{1} \\
\dot{p}_{1}=\mu_{1} q_{1}-q_{1}^{3}+\mu_{2}\left(q_{1}-q_{2}\right)-\left(q_{1}-q_{2}\right)^{3} \\
\dot{q}_{2}=p_{2} \\
\dot{p}_{2}=\mu_{3} q_{2}-q_{2}^{3}-\mu_{2}\left(q_{1}-q_{2}\right)+\left(q_{1}-q_{2}\right)^{3}
\end{gathered}
$$

where full details of the development of the simple smart structure model are provided by McInnes and Waters [18].

\section{Approximate heteroclinic connection and control}

We note that the system has been simplified by assuming $x / d \ll 1$. This approximation is sufficient to provide the required qualitative behaviour of the system through the Eq. (3), which is a simple cubic nonlinearity, while avoiding undue algebraic complexity. It will be shown that the system defined by Eqs. (13-16) has a number of equilibria which are both stable and unstable and may be connected through paths in the phase space of the problem. One type of path is the heteroclinic connection which requires the stable and unstable manifolds of two unstable equilibria to be connected. Solving Eqs. (14) and (16) for equilibrium conditions yields five equilibria for the parameter set, $\mu_{1}=1, \mu_{2}=1.5$ and $\mu_{3}=1$. The location of the equilibria are listed in Table 1 . The linear stability of these equilibria can be determined through linearisation of Hamilton's equations in the neighbourhood of each equilibrium point to determine the eigenvalues of the equilibria $\lambda_{j}(j=1-4)$. A set of stable equilibria are expected 
with conjugate imaginary eigenvalues and a set of unstable equilibria are expected with real eigenvalues of opposite sign [21]. It can then be determined that the 2 degree-of-freedom system possesses 3 unstable equilibria $E_{0}, E_{1}, E_{2}$ and 2 stable equilibria $E_{3}$ and $E_{4}$ shown in Fig. 3 with contours of potential $V$.

Table 1. Stability properties of the 5 equilibria of 2 degree-of-freedom bucking beam model[10].

\begin{tabular}{ccccccc}
\hline Point & $\tilde{q}_{1}$ & $\tilde{q}_{2}$ & $\lambda_{1,2}$ & $\lambda_{3,4}$ & $V$ & Type \\
\hline$E_{0}$ & 0 & 0 & \pm 1 & \pm 2 & 0 & Saddle $\times$ Saddle \\
$E_{1}$ & 1 & 1 & $\pm \sqrt{2} \mathrm{i}$ & \pm 1 & -0.5 & Saddle $\times$ Centre \\
$E_{2}$ & -1 & -1 & $\pm \sqrt{2} \mathrm{i}$ & \pm 1 & -0.5 & Saddle $\times$ Centre \\
$E_{3}$ & $-2 / 3$ & $2 / 3$ & $\pm 1 / \sqrt{3} \mathrm{i}$ & $\pm 2 \sqrt{2} \mathrm{i}$ & $-8 / 9$ & Saddle $\times$ Saddle \\
$E_{4}$ & $2 / 3$ & $-2 / 3$ & $\pm 1 / \sqrt{3} \mathrm{i}$ & $\pm 2 \sqrt{2} \mathrm{i}$ & $-8 / 9$ & Saddle $\times$ Saddle \\
\hline
\end{tabular}

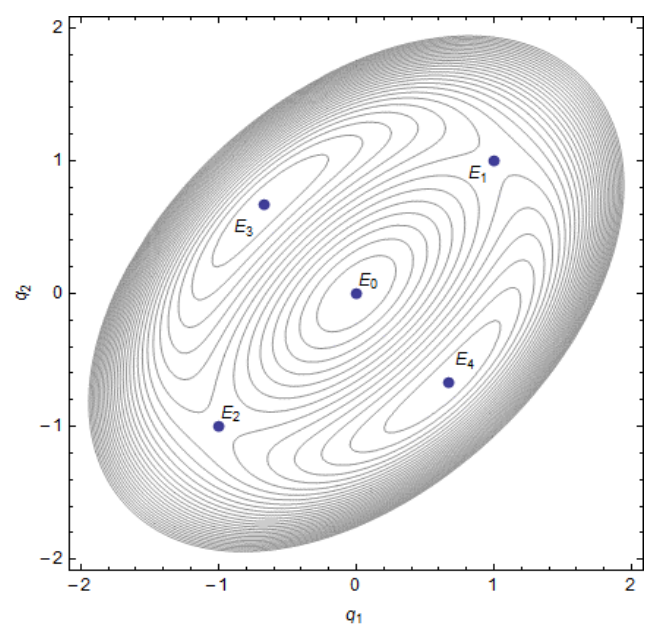

Figure 3. Potential $V(\boldsymbol{q}, \boldsymbol{\mu})$ and equilibria (3 unstable equilibria $E_{0}, E_{1}$ and $E_{2}$, and 2 stable equilibria $E_{3}$ and $\left.E_{4}\right)$.

Since the $E_{1}$ and $E_{2}$ lie on the same energy surface, there may be a heteroclinic connection connecting these equilibria, as shown in Fig. 4a, so that the structure can be reconfigured between these two equilibria without work being done. Similarly, if the structure is at the stable equilibria $E_{3}$, it needs to cross the potential barrier at $E_{1}$ to transition to the other stable equilibrium $E_{4}$, as shown in Fig. $4 \mathrm{~b}$, however energy must be added to the system to reach the top of the barrier and then dissipated to reach the final equilibrium state. 


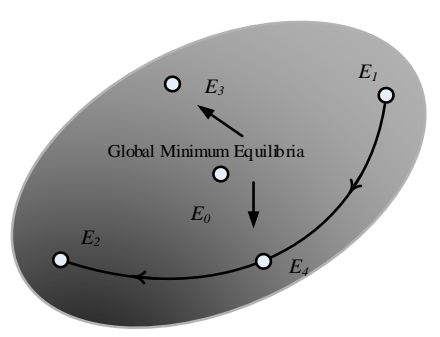

a

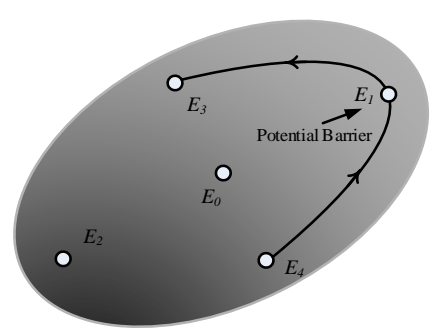

b

Figure 4. Transitions between different equilibria. (a) Ideal heteroclinic connection through $E_{3}$ or $E_{4}$ between $E_{1}$ and $E_{2}$, (b) Crossing the potential barrier $E_{1}$ between $E_{3}$ and $E_{4}$.

\subsection{Constructing the reference polynomial}

Although heteroclinic connections are essential characteristic of non-linear dynamical systems, it can be difficult to find exact heteroclinic connections numerically in complex nonlinear dynamical systems. Therefore, a method has been investigated to approximate heteroclinic connections which we envisage can form the basis for real-time reconfiguration of smart structures. The heteroclinic connection will be defined as a $4^{\text {th }}$ order polynomial, viz

$$
\boldsymbol{q}^{*}(t)=\boldsymbol{a}_{0}+\boldsymbol{a}_{1} t+\boldsymbol{a}_{2} t^{2}+\boldsymbol{a}_{3} t^{3}+\boldsymbol{a}_{4} t^{4}
$$

The unknown vector of constants $\boldsymbol{a}_{\mathrm{i}}(i=1-4)$ in the reference polynomial can then be related to the boundary conditions of the system.

The ideal heteroclinic connection in Fig. 4 departs from equilibrium $E_{1}$, goes through the global minimum at equilibrium $E_{4}$ and ends in equilibrium $E_{2}$. We can therefore define conditions on the polynomials which approximate the heteroclinic connection, viz

$$
\left[\begin{array}{lllll}
\boldsymbol{q}^{*}(0) & \boldsymbol{q}^{*}(T / 2) & \boldsymbol{q}^{*}(\mathrm{~T}) & \dot{\boldsymbol{q}}^{*}(0) & \dot{\boldsymbol{q}}^{*}(\mathrm{~T})
\end{array}\right]=\left[\begin{array}{cc}
1 & 1 \\
2 / 3 & -2 / 3 \\
-1 & -1 \\
0 & 0 \\
0 & 0
\end{array}\right]^{\mathrm{T}}
$$

Then, the only remaining free parameter to define the reference polynomial is the total reconfiguration duration $T$. Therefore, we can obtain an approximate heteroclinic connection defined using Eqs. (18) where the constant vectors of Eqs. (17) are found to be

$$
\left[\begin{array}{lllll}
\boldsymbol{a}_{0} & \boldsymbol{a}_{1} & \boldsymbol{a}_{2} & \boldsymbol{a}_{3} & \boldsymbol{a}_{4}
\end{array}\right]=\left[\begin{array}{cc}
1 & 1 \\
0 & 0 \\
14 / 3 T^{2} & -50 / 3 T^{2} \\
-52 / 3 T^{3} & 76 / 3 T^{3} \\
32 / 3 T^{4} & -32 / 3 T^{4}
\end{array}\right]^{\mathrm{T}}
$$


This function provides a smooth reference trajectory while ensuring that the required boundary conditions are satisfied. After repeated differentiation these polynomials provide the corresponding velocities and accelerations to be tracked to follow the reference trajectory.

Similarly, the transition from $E_{3}$ to $E_{4}$ can also be defined. We consider that this path should cross the potential barrier $E_{1}$, so the boundary conditions are defined as

$$
\left[\begin{array}{lllll}
\boldsymbol{q}^{*}(0) & \boldsymbol{q}^{*}(T / 2) & \boldsymbol{q}^{*}(\mathrm{~T}) & \dot{\boldsymbol{q}}^{*}(0) & \dot{\boldsymbol{q}}^{*}(\mathrm{~T})
\end{array}\right]=\left[\begin{array}{cc}
-2 / 3 & 2 / 3 \\
1 & 1 \\
2 / 3 & -2 / 3 \\
0 & 0 \\
0 & 0
\end{array}\right]^{\mathrm{T}}
$$

The constants of Eqs. (17) are therefore defined as:

$$
\left[\begin{array}{lllll}
\boldsymbol{a}_{\mathbf{0}} & \boldsymbol{a}_{1} & \boldsymbol{a}_{2} & \boldsymbol{a}_{3} & \boldsymbol{a}_{4}
\end{array}\right]=\left[\begin{array}{cc}
-2 / 3 & 2 / 3 \\
0 & 0 \\
20 / T^{2} & 12 / T^{2} \\
-104 / 3 T^{3} & -88 / 3 T^{3} \\
16 / T^{4} & 16 / T^{4}
\end{array}\right]^{\mathrm{T}}
$$

Now that the reference polynomials have been defined, an inverse method will be developed in order to track them, allowing an approximate heteroclinic connection to be followed.

\subsection{Inverse methods}

Inverse control allows tracking of time dependent constrains and is an effective method to control nonlinear systems, used extensively in a diverse range of nonlinear control problems [22]. A nonlinear system is assumed to have a generic form of

$$
\dot{\boldsymbol{x}}(t)=f\{\boldsymbol{x}(t), \boldsymbol{u}(t) ; t\}, \boldsymbol{x} \in R^{m}, \boldsymbol{u} \in R^{n}, t \in[0, T]
$$

where $\boldsymbol{x}(t)$ is the system state, $\boldsymbol{u}(t)$ is a vector of inputs and $f$ is a smooth function describing the dynamics of the process. The generic boundary conditions and constrains are defined as

$$
x(0)=x_{0}, x(T)=x_{f}
$$

The inverse method represents the control problem of how to find a control vector $\boldsymbol{u}(t)$ which can track desired outputs of the system while meeting the requirements of the boundary conditions so that

$$
\boldsymbol{e}\left\{\boldsymbol{x}(t), \boldsymbol{x}^{*}(t) ; t\right\}=\left\{\boldsymbol{x}(t)-\boldsymbol{x}^{*}(t)\right\}=0
$$

where $\boldsymbol{e}$ is a continuous constraint function and $\boldsymbol{x}^{*}(t)$ represents the desired output. This relationship should be differentiated repeatedly until the control vector appears explicitly.

For our dynamical system we need to extend this method to provide nonlinear control to track the reference trajectory in the presence of uncertainties. We may differentiate the constraint vector $\boldsymbol{e}$ until 
the control appears explicitly, then we may add feedback terms instead of defining the constraint vector to be null so that

$$
\ddot{\boldsymbol{e}}\left\{\boldsymbol{x}(t), \boldsymbol{x}^{*}(t) ; t\right\}=-\boldsymbol{g}_{1} \dot{\boldsymbol{e}}-\boldsymbol{g}_{2} \boldsymbol{e}
$$

where $\boldsymbol{g}_{1}$ and $\boldsymbol{g}_{2}$ are constant gain matrices given by

$$
\begin{aligned}
& \boldsymbol{g}_{1}=\operatorname{Diag}\left\{g_{11}, g_{12}\right\} \\
& \boldsymbol{g}_{2}=\operatorname{Diag}\left\{g_{21}, g_{22}\right\}
\end{aligned}
$$

The $4^{\text {th }}$ order polynomial can then be used as a reference trajectory with the inverse control method to provide an example of a controlled heteroclinic connection through $E_{3}$ between $E_{1}$ and $E_{2}$, with $\mu_{1}=1$, $\mu_{2}=1.5$ and $\mu_{3}=1$ as parameters, and the reconfiguration duration later set as $T=20$.

Recall Eq. (12), (13), (14) and (15), which can be expressed in matrix form as

$$
\left[\begin{array}{c}
\dot{q}_{1} \\
\dot{p}_{1} \\
\dot{q}_{2} \\
\dot{p}_{2}
\end{array}\right]=\left[\begin{array}{c}
p_{1} \\
-q_{1}^{3}-\left(q_{1}-q_{2}\right)^{3} \\
p_{2} \\
-q_{2}^{3}+\left(q_{1}-q_{2}\right)^{3}
\end{array}\right]+\left[\begin{array}{ccc}
0 & 0 & 0 \\
q_{1} & \left(q_{1}-q_{2}\right) & 0 \\
0 & 0 & 0 \\
0 & -\left(q_{1}-q_{2}\right) & q_{2}
\end{array}\right]\left[\begin{array}{l}
\mu_{1} \\
\mu_{2} \\
\mu_{3}
\end{array}\right]
$$

This is now in the form of $\dot{\boldsymbol{x}}=f(\boldsymbol{x})+h(\boldsymbol{x}) \boldsymbol{u}$, which is an affine system with drift terms, where $\dot{\boldsymbol{x}}$ is a vector of state variables and $\boldsymbol{u}$ is a vector of control variables. Feedback linearisation can then be used to transform the original system model into an equivalent linear model, by algebraically transforming the nonlinear system into linear dynamics, so that linear control methods can be applied. Feedback linearisation therefor uses exact feedback, while conventional (Jacobian) linearisation which is a linear approximation of the actual non-linear dynamics [23].

We can rewrite Eq. (26) in a simpler form:

$$
\begin{aligned}
{[\ddot{\boldsymbol{q}}]=\left[\begin{array}{l}
-q_{1}^{3}-\left(q_{1}-q_{2}\right)^{3} \\
-q_{2}^{3}+\left(q_{1}-q_{2}\right)^{3}
\end{array}\right]+J(x) \boldsymbol{\mu} } \\
\quad=\left[\begin{array}{l}
-q_{1}^{3}-\left(q_{1}-q_{2}\right)^{3} \\
-q_{2}^{3}+\left(q_{1}-q_{2}\right)^{3}
\end{array}\right]+\left[\begin{array}{ccc}
q_{1} & \left(q_{1}-q_{2}\right) & 0 \\
0 & -\left(q_{1}-q_{2}\right) & q_{2}
\end{array}\right]\left[\begin{array}{l}
\mu_{1} \\
\mu_{2} \\
\mu_{3}
\end{array}\right]
\end{aligned}
$$

The invertibility matrix: $J(x)$ has rank 2 when there are two values not equal to zero among the three variables $q_{1}, q_{2}$ and $q_{1}-q_{2}$. From Section 3.1, the control system will then have four null points, when $q_{1}-q_{2}=0$ and $q_{1}=0$ and $q_{2}=0$. Therefore, the three control parameters can be chosen to avoid singularities, for example when $q_{1}$ vanishes, the corresponding value of $\mu_{1}$ will be unbound, so we can then select $\mu_{2}$ and $\mu_{3}$ as the control parameters with a constant value of $\mu_{1}$. The system is therefore controllable with two state variables and two control variables. In this way, the natural length of the spring can be used as the control (assumed through an appropriate smart material) and so the control parameters which can be used to track the reference trajectory are given by 


$$
\boldsymbol{\mu}=J^{-1}(x)\left(\ddot{\boldsymbol{q}}-\left[\begin{array}{l}
-q_{1}^{3}-\left(q_{1}-q_{2}\right)^{3} \\
-q_{2}^{3}+\left(q_{1}-q_{2}\right)^{3}
\end{array}\right]\right)
$$

In this paper we formulate a boundary value for the critical region of possible control. A simple algorithm is used to determine the singular states and then provide a new set of control variables. Here, $\delta$, which is defined in Section 2, is used as an evaluation index to achieve control. Although $\boldsymbol{\mu}$ is the nominal control vector, the real situation should be considered: the spring is elastic but within limits. Therefore the real deformation $\delta$ is defined to be no more than $25 \%$ to approximate a realistic system. Therefore, when $\delta_{2}$ is more than $\bar{\delta}$, where $\bar{\delta}$ corresponds to a deformation of $25 \%$, as defined through Eq. (6), $\mu_{1}$ and $\mu_{3}$ are chosen as the control variables with fixed $\mu_{2}$; when $\delta_{1}$ is more than $\bar{\delta}, \mu_{2}$ and $\mu_{3}$ are choose as the control variables with fixed $\mu_{1}$; when $\delta_{3}$ is more than $\bar{\delta}, \mu_{1}$ and $\mu_{2}$ are chosen as the control variables with fixed $\mu_{3}$, with Eq. (28) providing the control variables $\mu_{1}, \mu_{2}$ and $\mu_{3}$, where $\delta_{1}$, $\delta_{2}$ and $\delta_{3}$ represent the deformation ratio of real springs corresponding to $\mu_{1}, \mu_{2}$ and $\mu_{3}$, respectively. Using Eqs. (24) and (25), the constraint can then be modified as

$$
\begin{gathered}
\boldsymbol{e}=\boldsymbol{q}-\boldsymbol{q}^{*} \\
\dot{\boldsymbol{e}}=\dot{\boldsymbol{q}}-\dot{\boldsymbol{q}}^{*} \\
\ddot{\boldsymbol{e}}=\ddot{\boldsymbol{q}}-\ddot{\boldsymbol{q}}^{*}=-\boldsymbol{g}_{1} \dot{\boldsymbol{e}}-\boldsymbol{g}_{2} \boldsymbol{e}
\end{gathered}
$$

In order to ensure convergence to the desired output $\boldsymbol{q}^{*}$ a Lyapunov function will be defined as

$$
\phi(\boldsymbol{q}, \dot{\boldsymbol{q}})=\frac{1}{2}\left(\boldsymbol{q}-\boldsymbol{q}^{*}\right)^{T} \boldsymbol{g}_{2}\left(\boldsymbol{q}-\boldsymbol{q}^{*}\right)+\frac{1}{2}\left(\dot{\boldsymbol{q}}-\dot{\boldsymbol{q}}^{*}\right)^{T}\left(\dot{\boldsymbol{q}}-\dot{\boldsymbol{q}}^{*}\right)
$$

where $\phi(\boldsymbol{q}, \dot{\boldsymbol{q}})>0$ and $\phi\left(\boldsymbol{q}^{*}, \dot{\boldsymbol{q}}^{*}\right)=0$ for $\boldsymbol{g}_{2}>\mathbf{0}$. The time derivative of the Lyapunov function is clearly

$$
\dot{\phi}(\boldsymbol{q}, \dot{\boldsymbol{q}})=\left(\dot{\boldsymbol{q}}-\dot{\boldsymbol{q}}^{*}\right)^{T} \boldsymbol{g}_{2}\left(\boldsymbol{q}-\boldsymbol{q}^{*}\right)+\left(\dot{\boldsymbol{q}}-\dot{\boldsymbol{q}}^{*}\right)^{T}\left(\ddot{\boldsymbol{q}}-\ddot{\boldsymbol{q}}^{*}\right)=\dot{\boldsymbol{e}}^{T} \boldsymbol{g}_{2} \boldsymbol{e}+\dot{\boldsymbol{e}}^{T} \ddot{\boldsymbol{e}}
$$

Substituting Eq (31) into Eq (33) we obtain the expression of the time derivative of the Lyapunov function. It can be seen that $\phi$ is monotonically decreasing corresponding to $\boldsymbol{g}_{\mathbf{1}}>0$ and $\boldsymbol{g}_{\mathbf{2}}>0$ such that

$$
\dot{\phi}(q, \dot{q})=\dot{e}^{T} g_{2} e+\dot{e}^{T}\left(-g_{1} \dot{e}-g_{2} e\right)=-\dot{e}^{T} g_{1} \dot{e} \leq 0
$$

where again $g_{1}$ and $g_{2}$ are the gain matrix. It is then clear that the required acceleration is given by

$$
\ddot{q}_{1}=\ddot{q}_{1}^{*}-g_{11}\left(\dot{q}_{1}-\dot{q}_{1}^{*}\right)-g_{12}\left(q_{1}-q_{1}^{*}\right)
$$




$$
\ddot{q}_{2}=\ddot{q}_{2}{ }^{*}-g_{21}\left(\dot{q}_{2}-\dot{q}_{2}^{*}\right)-g_{22}\left(q_{2}-q_{2}^{*}\right)
$$

Through intermediate variables $\ddot{q}_{1}$ and $\ddot{q}_{2}$, the inverse control method can be connected to the system dynamics. That is, Eqs. (35) and (36) are used as feedback to control the dynamics of the system defined by Eqs. (13)-(16). The system dynamics are therefore artificially linearised about the nominal reference trajectory so that the control variables can then be determined from Eq. (28) as

$$
\boldsymbol{\mu}=J^{-1}(x)\left(\begin{array}{l}
-\lambda_{11} \dot{q}_{1} \\
-\lambda_{21} \dot{q}_{2}
\end{array}+\left[\begin{array}{l}
\ddot{q}_{1}{ }^{*}+g_{11} \dot{q}_{1}{ }^{*}-g_{12}\left(q_{1}-q_{1}{ }^{*}\right)+q_{1}^{3}+\left(q_{1}-q_{2}\right)^{3} \\
\ddot{q}_{2}{ }^{*}+g_{21} \dot{q}_{2}{ }^{*}-g_{22}\left(q_{2}-q_{2}{ }^{*}\right)+q_{2}^{3}-\left(q_{1}-q_{2}\right)^{3}
\end{array}\right]\right)
$$

This provides a composite control which ensures convergence to the desired reference trajectory while avoiding control singularities, as shown in Fig. 5. Equation (37) provides a distinct relationship between the control variables $\boldsymbol{\mu}$ and the state variables $\boldsymbol{q}$, so the state variables $\boldsymbol{q}$ form the control variables $\boldsymbol{\mu}$ along with the reference trajectory $\boldsymbol{q}^{*}$. For a practical implementation the actuator band width and measurement noise needed to be considered, however, this ideal smart structure model just focus on the mathematical theory and control manoeuvre, therefore, for future application, more details about the actuator and sensor need elaborate consideration.

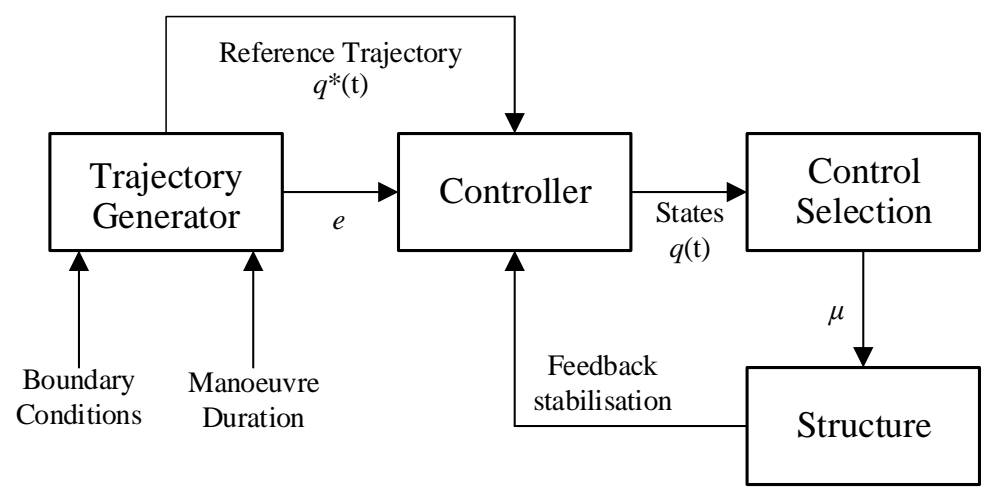

Figure 5. Composite feedback linerisation control scheme.

\subsection{Energy evaluation criteria}

In order to control the reconfiguration of the model smart structure we have implicitly assumed that the natural length of the springs can be modulated through the parameter set $\mu_{1}, \mu_{2}$ and $\mu_{3}$ (again, assuming use of an appropriate smart material, such as shape-memory alloys which can be deformed when heated). In order to estimate the energy requirements for such modulation we provide a simplified description of the spring actuator [24], as presented in Fig. 6. Two performance parameters should be considered in the model, one is the basic property of the smart material, the induced-strain effect, denoted by $d_{s}$ in Fig. 6; the other is the internal stiffness, $k_{s}$, again shown in Fig.6. Due to spring compressibility, an elastic displacement $F / k_{S}$ can be produced by the load $F$. The spring can actuate the induced-strain 
displacement, $d_{\mathrm{s}}$, to increase or decrease the output displacement $d_{e}$, as shown in Fig.6a, where $d_{e}$ is given as

$$
d_{e}=d_{s}-\frac{F}{k_{s}}
$$

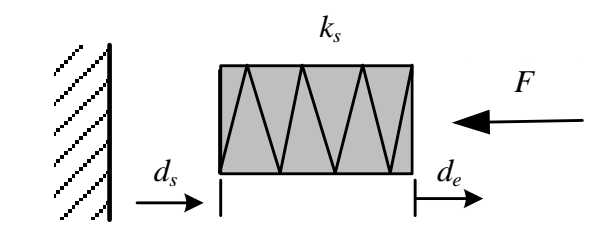

a

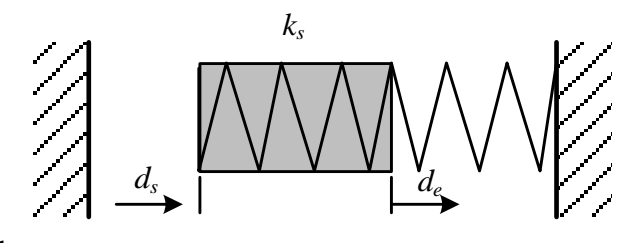

$\mathrm{b}$

Figure 6. Control effort evaluation criteria using a simple spring model. Shaded block represents smart material element with internal stiffness. (a) Element under external load $F$. (b) Element attached to external spring (adapted from [23]).

Now the external load, $F$, is considered as a product of an external spring with same stiffness $k_{S}$, as shown in Fig.6b, thus

$$
F=k_{s} d_{e}
$$

Combing Eq. (38) and Eq.(39), the relationship between $d_{e}$ and $d_{s}$ can be found as

$$
d_{s}=2 d_{e}
$$

Under quasi-static conditions, the output energy is then half the product between the force and the output displacement, i.e.

$$
E=\frac{1}{2} k_{s} d_{e}^{2}
$$

Substituting Eq. (40) into Eq. (41) we obtain the expression for input energy in terms of induced-strain, as

$$
E=\frac{1}{2} k_{S}\left(\frac{1}{4} d_{S}^{2}\right)
$$

Now we consider the relationship between the energy input and control action more specifically. Through the above analysis, and from Section 2 , we can use the control variable $\mu=2(l / d-1)$ to obtain

$$
\Delta l=(d / 2) \Delta \mu
$$

Consider that $\Delta l$ and $d_{s}$ have the same significance in the spring model so that Eq. (41) may be written as 


$$
E=\frac{1}{2} k_{S}\left(\frac{1}{4} d_{S}^{2}\right)=\frac{1}{2} k_{S}\left(\frac{1}{4}\left(\frac{d}{2}\right)^{2} \Delta \mu^{2}\right)=\frac{1}{32} k_{S} d^{2} \Delta \mu^{2} \propto \Delta \mu^{2}
$$

where $k_{s}, d$ are constants. Using Eq. (44) we now have a simple means of comparting the energy requirements to track the reference polynomial trajectory between equilibrium states of the smart structure model.

\subsection{Numerical solutions}

The method defined in Section 3.2 will now be applied to illustrate two reconfiguration manoeuvres and the use of the inverse method to achieve effective control. The inverse method will be used to reconfigure the 2 degree-of-freedom beam model with a $4^{\text {th }}$ order polynomial to approximate the ideal heteroclinic connection through $E_{3}$ between $E_{1}$ and $E_{2}$. The approximate heteroclinic connection can be seen in Fig. 7a, where the controller tracks the approximate trajectory defined by the $4^{\text {th }}$ order polynomial, with the constant gains defined as $g_{11}=g_{21}=0.25$ and $g_{12}=g_{22}=0.75$ and the reconfiguration duration $T=20$. The corresponding shape of the structure during the transition from $E_{1}$ to $E_{2}$ is shown in Fig. $7 \mathrm{~b}$. The labels in Fig. $7 \mathrm{~b}$ illustrate the transition process corresponding to the positions marked in Fig. 7a. The corresponding controls $\mu_{1}, \mu_{2}$ and $\mu_{3}$ are shown in Fig. 7c, where it can be seen that the controls are symmetric about $t=T / 2$ as expected. The controls here are scaled variables, so that although the maximum relative change shown in the Fig. $7 \mathrm{c}$ is more than $50 \%$, the ratio of the corresponding real spring deformation is only 18\%, which is calculated by Eq.(6), and is less than $\bar{\delta}$. There are sudden jumps in Fig. 7c which correspond to the switching control strategy discussed in section 3.2.

The corresponding mass displacement and the reference path is then shown in Fig. $7 d$. 


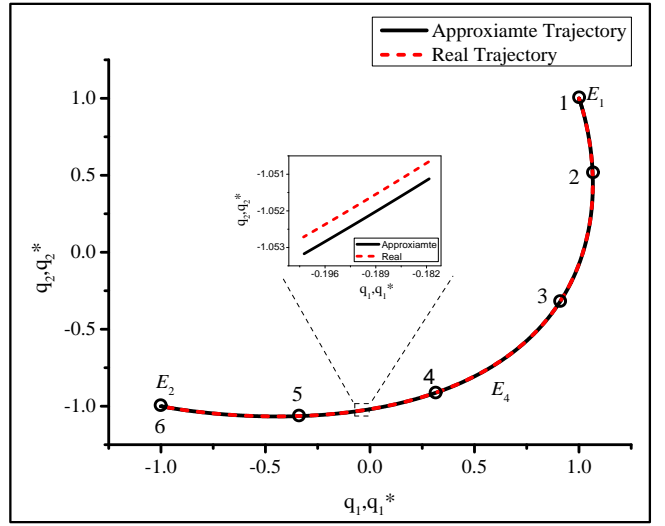

a

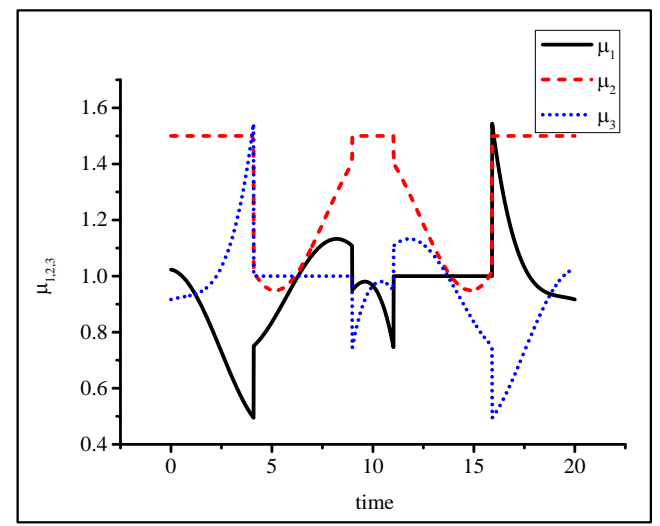

$\mathrm{C}$
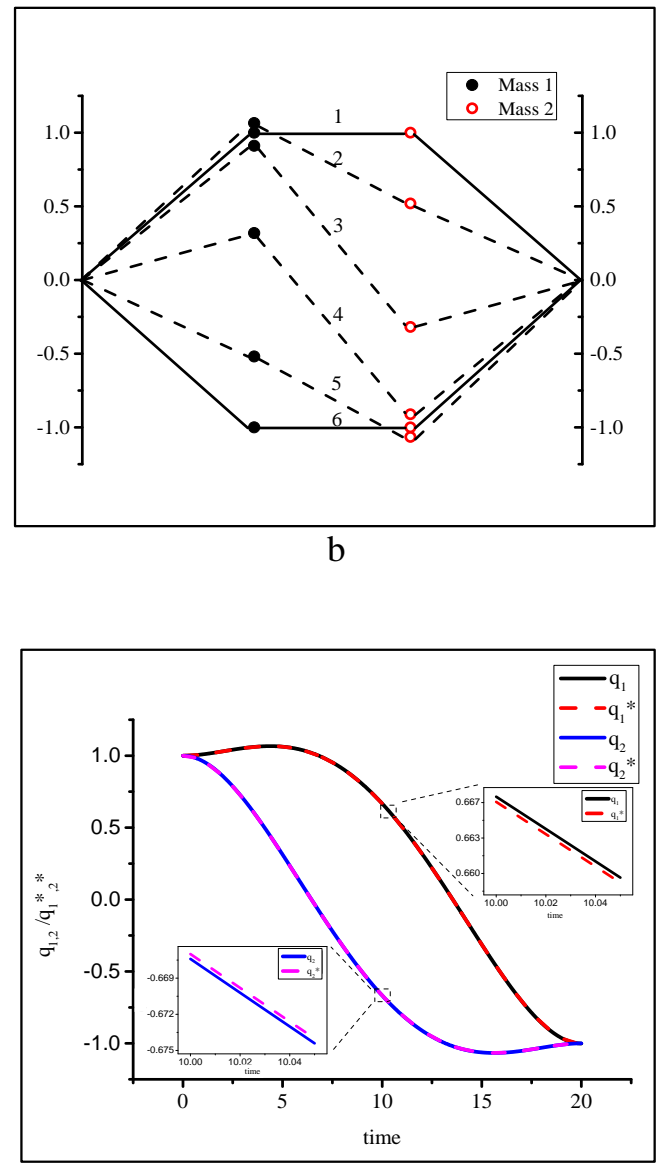

$\mathrm{d}$

Figure 7. $4^{\text {th }}$ order polynomial as reference trajectory from $E_{1}$ at $(1,1)$ to $E_{2}$ at $(-1,-1)$. (a) Controlled transition, (b) Geometry of transition process, (c) Controls actuated through the parameters $\mu_{1}, \mu_{2}$ and $\mu_{3}$, (d) Mass displacements during the transition from $E_{1}$ to $E_{2}$ with the reference trajectory and actual trajectory.

We now consider the inverse method to reconfigure the 2 degree-of-freedom beam model with the $4^{\text {th }}$ order polynomial to approximate a transition crossing the potential barrier $E_{1}$ between $E_{3}$ and $E_{4}$. The approximate path can be seen in Fig. 8(a) and the corresponding shape of the structure during the transition from $E_{3}$ to $E_{4}$ is shown in Fig. 8(b). The corresponding controls $\mu_{1}, \mu_{2}$ and $\mu_{3}$ are shown in Fig. 8(c) and the corresponding mass displacement and the reference path shown in Fig. 8(d).

We now use the evaluation criteria discussed in Section 3.3 to investigate the energy requirements of the transitions of the simple smart structure model. For example, the energy requirement needed to overcome the potential barrier at equilibrium $E_{1}$ is clearly greater than that passing through $E_{3}$, as shown in Fig. 9. The energy for transitions through $E_{1}$ is of order $25 \%$ higher than that for transitions through $E_{3}$, as expected. 


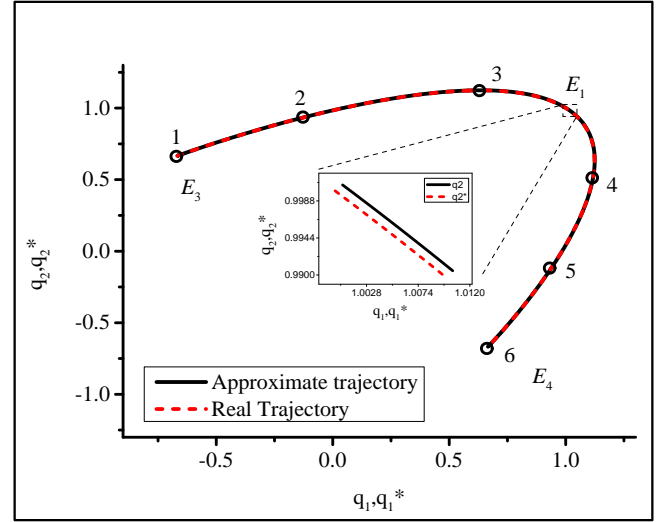

a

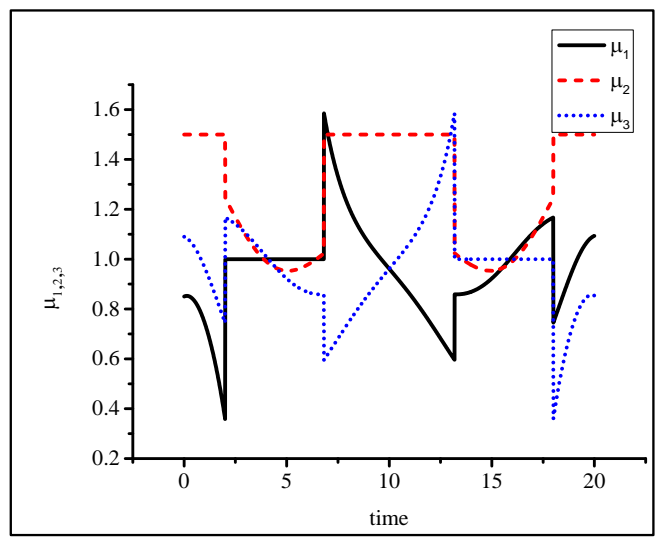

c
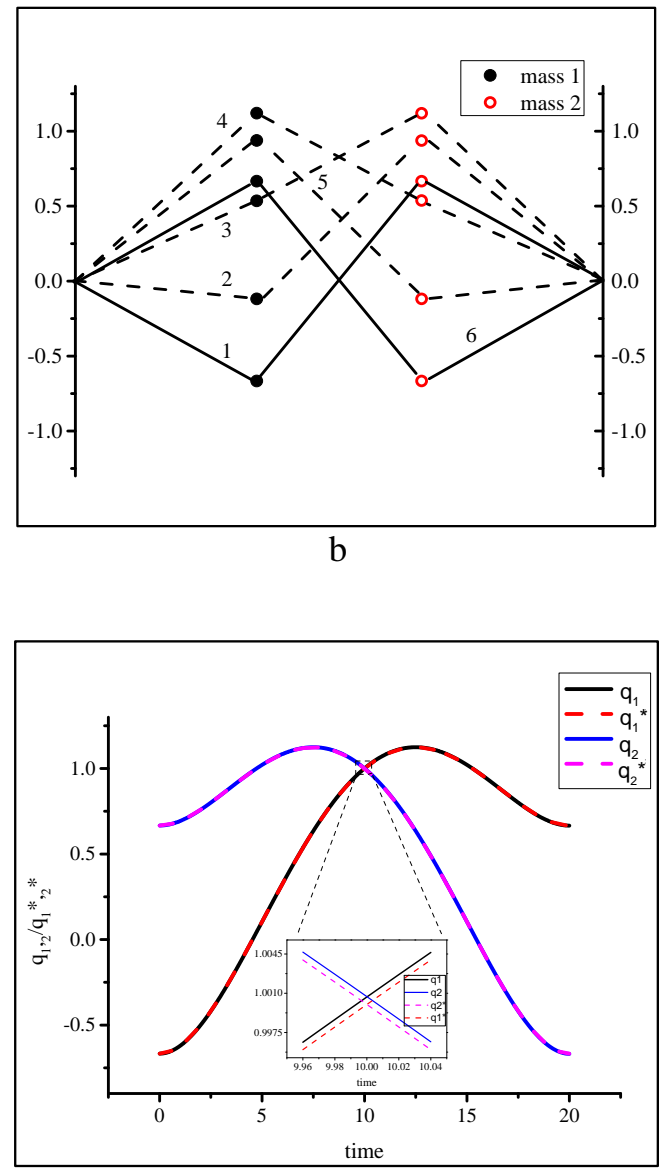

d

Figure 8. $4^{\text {th }}$ order polynomial reference trajectory as reference trajectory from $E_{3}$ at $(-2 / 3,2 / 3)$ to $E_{4}$ at $(2 / 3,-2 / 3)$. (a) Controlled transition, (b) Geometry of transition process, (c) Controls actuated through the parameters $\mu_{1}, \mu_{2}$ and $\mu_{3}$, (d) Mass displacements during the transition from $E_{3}$ to $E_{4}$ with the reference trajectory and actual trajectory.

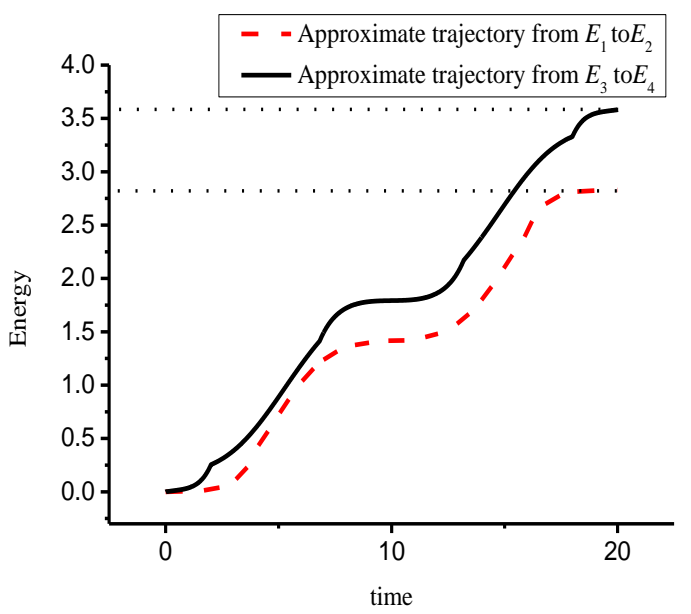

Figure 9. Comparison of energy input for different reconfigurations.

3.5. Extending the order of the polynomial 
In order to evaluate the polynomial method further, a set of higher order polynomials can be used which can reduce the effective energy required for reconfiguration. We can therefore add additional boundary conditions to construct a higher order reference polynomial. Considering the transition from $E_{1}$ at $(1,1)$ to $E_{2}$ at $(-1,-1)$ as an example we define

$$
\left[\ddot{\boldsymbol{q}}^{*}(0) \quad \ddot{\boldsymbol{q}}^{*}(\mathrm{~T})\right]=\left[\begin{array}{ll}
0 & 0 \\
0 & 0
\end{array}\right]
$$

Then, the only remaining free parameter to define the reference polynomial is again the total reconfiguration duration $T$. We can therefore obtain an approximate heteroclinic connection defined using

$$
\boldsymbol{q}^{*}(t)=\boldsymbol{a}_{0}+\boldsymbol{a}_{1} t+\boldsymbol{a}_{2} t^{2}+\boldsymbol{a}_{3} t^{3}+\boldsymbol{a}_{4} t^{4}+\boldsymbol{a}_{5} t^{5}+\boldsymbol{a}_{6} t^{6}
$$

Using the inverse control method we can generate another approximate heteroclinic connection as shown in Fig. 10(a), where the controller tracks the approximate trajectory defined by the $6^{\text {th }}$ order polynomial. The corresponding controls $\mu_{1}, \mu_{2}$ and $\mu_{3}$ are shown in Fig. 10(b).

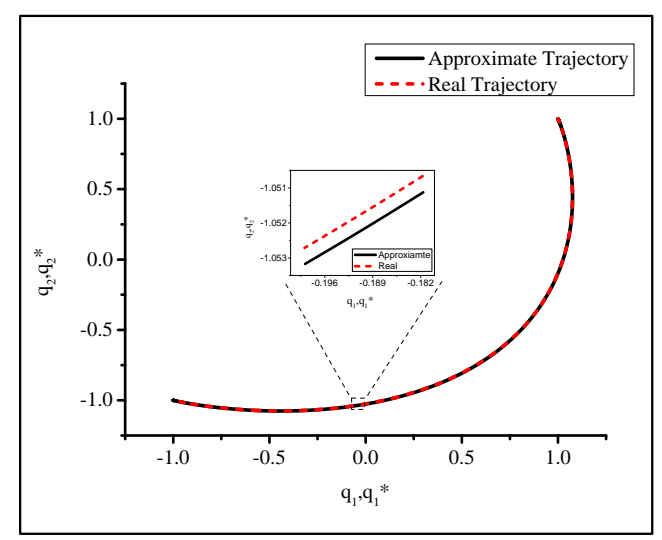

a

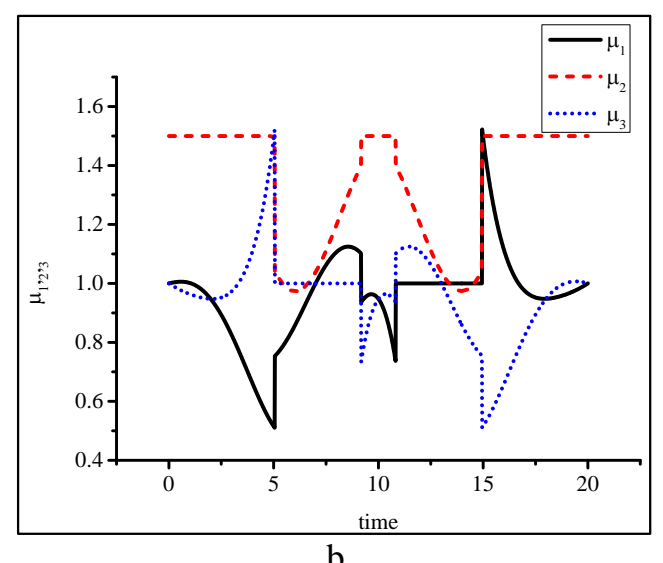

Figure 10. $6^{\text {th }}$ order polynomial as reference trajectory from $E_{1}$ at $(1,1)$ to $E_{2}$ at $(-1,-1)$. (a) Controlled transition, (b) Controls actuated through the parameters $\mu_{1}, \mu_{2}$ and $\mu_{3}$.

Then, we can use the energy evaluation criteria in order to track the approximate trajectory through $E_{3}$, where the total energy input to the process can be seen in Fig. 11. The numerical results demonstrate that with the higher order polynomial less energy is required for the reconfiguration process. 


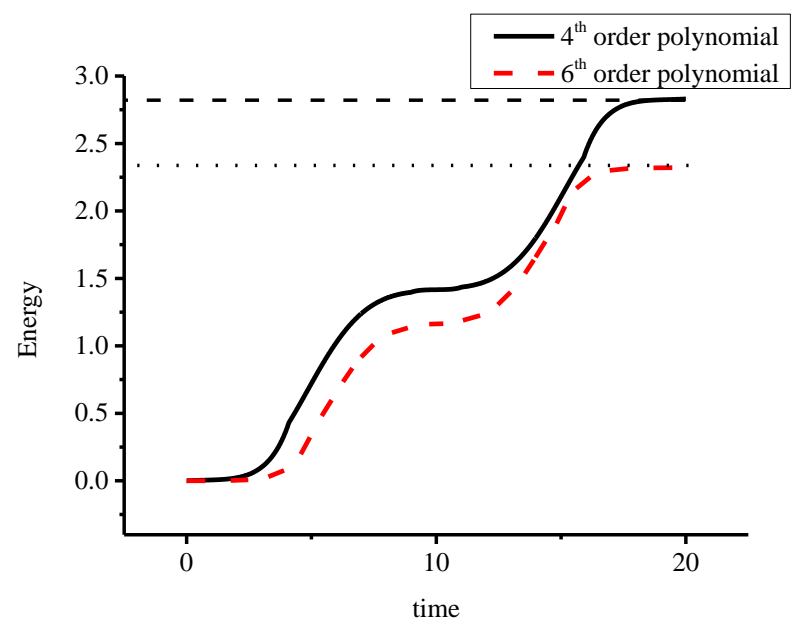

Figure 11. Comparison of energy input to track different approximate trajectories.

We now add additional target waypoints so that we can use these points to construct a more accurate polynomial to approximate the exact heteroclinic connection. Here, we will use the energy evaluation criteria and an optimisation algorithm to find the location of these waypoints to minimise the total energy required. Additional variables will be added based on Eq. (46), as shown in Eq. (47) so that

$$
\boldsymbol{q}^{*}(t)=\boldsymbol{a}_{0}+\boldsymbol{a}_{1} t+\boldsymbol{a}_{2} t^{2}+\boldsymbol{a}_{3} t^{3}+\boldsymbol{a}_{4} t^{4}+\boldsymbol{a}_{5} t^{5}+\boldsymbol{a}_{6} t^{6}+\boldsymbol{a}_{7} t^{7}+\boldsymbol{a}_{8} t^{8}
$$

It is difficult to add additional constant $\boldsymbol{a}_{7}$ and $\boldsymbol{a}_{8}$ from simple geometric considerations. However, two time points $T / 4$ and $3 T / 4$ (where $T$ is again the reconfiguration duration) are selected as fixed waypoints. Then, the location of the two target waypoints are chosen by using an optimisation algorithm. Therefore, an $8^{\text {th }}$ order polynomial can be defined as the reference trajectory and we use the fmincon function in Matlab, which is a nonlinear multivariable optimiser which can find the minimum of a constrained function [25]. We would envisage using a simple numerical search algorithm to optimise the waypoint location in a real smart structure using heteroclinic connections. Using the optimisation algorithm, the improved numerical results can be seen in Fig. 12(a), showing the heteroclinic connection approximated with an $8^{\text {th }}$ order polynomial where the constant gain matrices are again $g_{11}=g_{21}=0.25, g_{12}=$ $g_{22}=0.75$. Figure 12(b) illustrates the corresponding shape of the structure during the transition from $E_{1}$ to $E_{2}$ and the corresponding controls $\mu_{1}, \mu_{2}$ are $\mu_{3}$ are shown in Fig. 12(c). It can be seen that the controls are again symmetric about $t=T / 2$. The corresponding mass displacement and the reference path is shown in Fig. 12(d). 

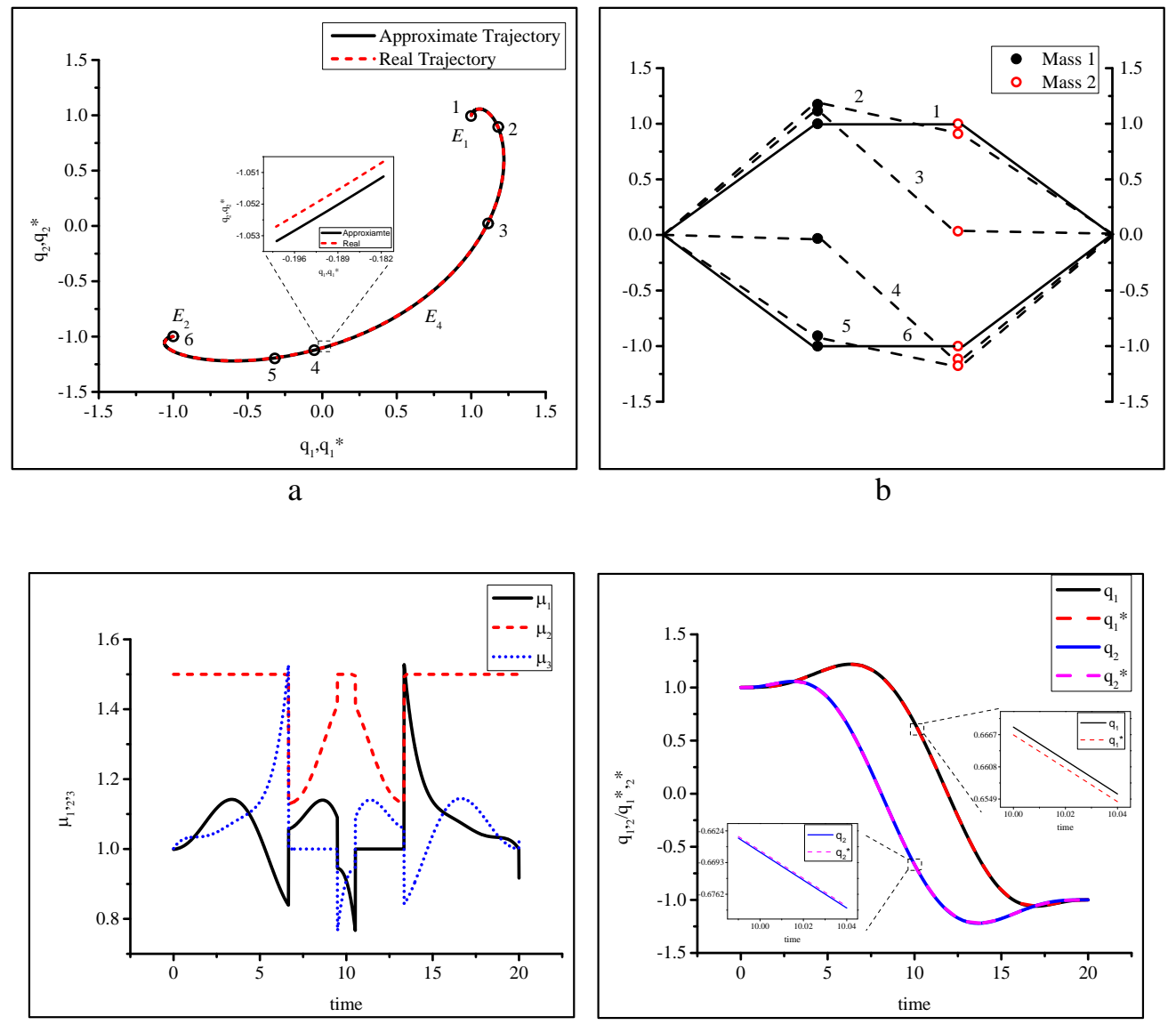

$\mathrm{c}$

d

Figure 12. $8^{\text {th }}$ order polynomials as reference trajectory from $E_{1}$ at $(1,1)$ to $E_{2}$ at $(-1,-1)$. (a) Controlled transition, (b) Geometry of transition process, (c) Controls actuated through the parameters $\mu_{1}, \mu_{2}$ and $\mu_{3}$, (d) Mass displacements during the transition from $E_{1}$ to $E_{2}$ with reference trajectory and actual trajectory.

The energy evaluation criteria can then be used to measure the total energy required for the reconfiguration process, as can be seen in Fig. 13. From Fig. 13 it can be seen that the initial assumptions on the order of the polynomial which is used to approximate the heteroclinic connection is key. We can use a higher order polynomial as a reference trajectory to reconfigure structure with significantly less energy input, but requiring a numerical search for optimisation. 


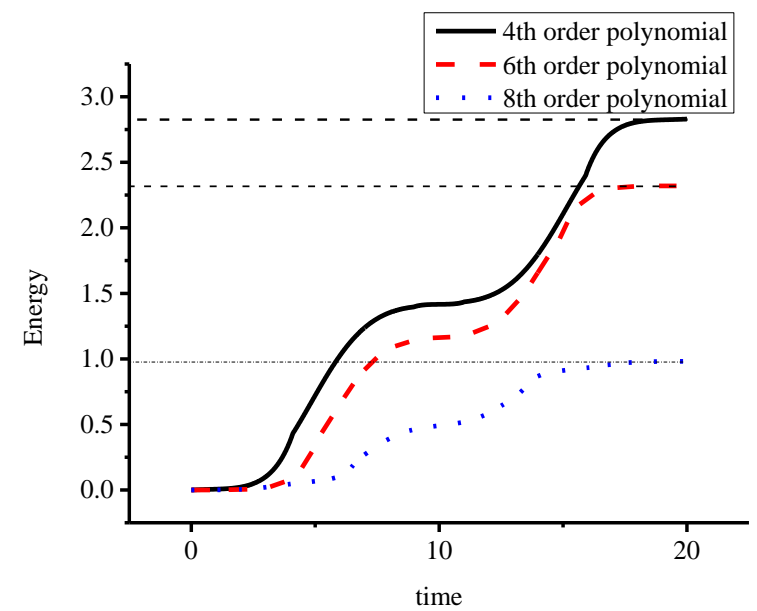

Figure 13. Comparison of energy input to track different approximate trajectories with varying polynomial degree.

Now, we will consider the influence of the total reconfiguration duration $T$, which is the only remaining free parameter to define the reference polynomials. Using the same energy evaluation criteria we can find the relationship between total reconfiguration duration and the energy requirements. Figure 14 shows five distinct curves which define five types of reference trajectory with different manoeuvre durations considered. There is an evident sharp decrease to an optimum, minimum energy duration and then a slow increase as the manoeuvre duration grows. For this example we can therefore identify the optimum manoeuvre duration $T$. It can again be seen that the transition through $E_{1}$ needs more energy than the transition through $E_{3}$ with the same order polynomial and the same manoeuvre duration as expected. This demonstrates that the higher order polynomial can significantly improve the reference trajectory for reconfiguring the smart structure model.

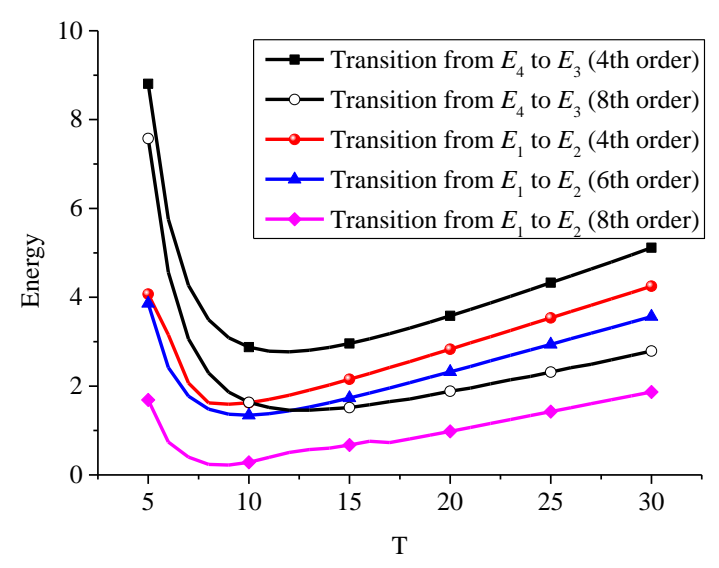

Figure 14. Energy required for reconfiguration as a function of reconfiguration duration.

\section{Three mass chain}

4.1.3 mass chain model 
In order to further explore the possibility of reconfiguring smart structures using reference polynomials, a more complex three mass chain with four linear springs will now be considered, with the springs clamped at both ends as shown in Fig. 15. This more complex problem greatly increases the number of equilibria in the system and the difficulty of finding an exact heteroclinic connection by purely numerical means. The dynamics of the chain are firstly obtained from Eqs. (2-3) as

$$
\begin{aligned}
& \dot{q}_{1}=p_{1} \\
& \dot{p_{1}}=\mu_{1} q_{1}-q_{1}^{3}+\mu_{2}\left(q_{1}-q_{2}\right)-\left(q_{1}-q_{2}\right)^{3} \\
& \dot{q}_{2}=p_{2} \\
& \dot{p_{2}}=\mu_{3}\left(q_{2}-q_{3}\right)-\left(q_{2}-q_{3}\right)^{3}-\mu_{2}\left(q_{1}-q_{2}\right)+\left(q_{1}-q_{2}\right)^{3} \\
& \dot{q}_{3}=p_{3} \\
& \dot{p_{3}}=\mu_{4} q_{3}-q_{3}^{3}-\mu_{3}\left(q_{2}-q_{3}\right)+\left(q_{2}-q_{3}\right)^{3}
\end{aligned}
$$

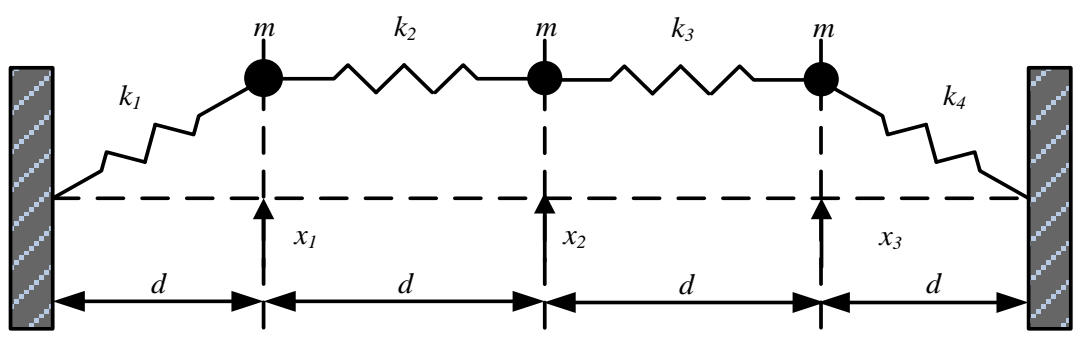

Figure 15. 3 degree-of-freedom bucking beam model.

Solving Eqs. (48-53) for equilibria, the location of the equilibria can be found as shown in Table 2 for the parameter set, $\mu_{1}=1, \mu_{2}=1.5, \mu_{3}=1.5$ and $\mu_{4}=1$ along with the eigenvalue spectrum associated with each equilibrium, as discussed in Section 3. It can be seen from Table 2 that the system possesses 1 unstable equilibrium $E_{0}$, where the potential has a global maximum; 20 unstable equilibria where the potential has several saddles, and 6 stable equilibria where the potential has a local minimum. The location of these equilibria and potential surfaces can be seen in Fig.16 and Fig.17, respectively. 


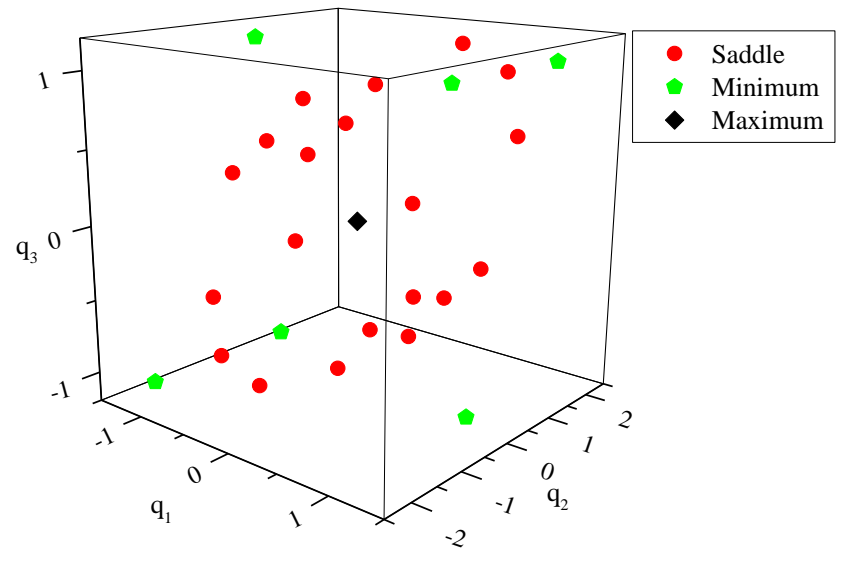

Figure 16. 27 equilibria (21 unstable equilibria and 6 stable equilibria)
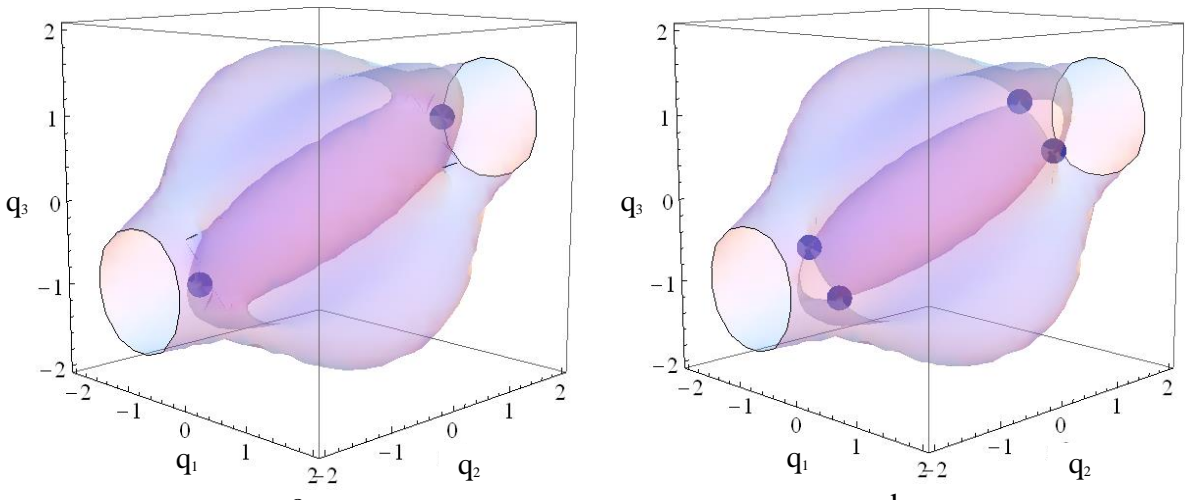

$\mathrm{b}$
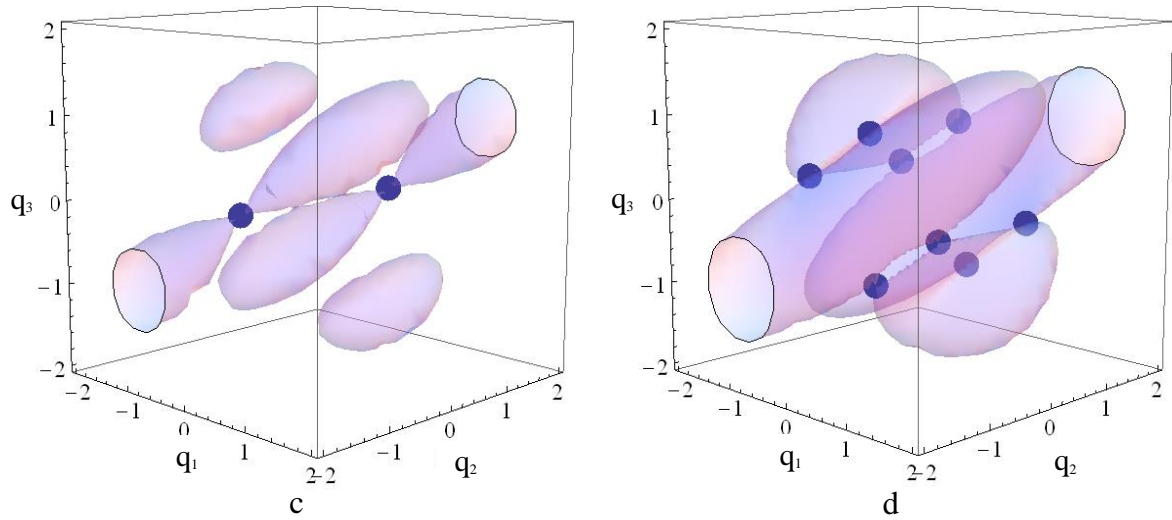

Figure 17. Potential contour with Saddles. (a) Potential -0.5 with $E_{1}$ and $E_{2}$, (b) Potential -0.482 with $E_{23}$ to $E_{26}$, (c) Potential -1.125 with $E_{3}$ and $E_{4}$, (d) Potential -0.844 with $E_{11}$ and $E_{22}$. 
Table 2. Stability properties of the 27 equilibria of a three mass chain with $\mu_{1}=1, \mu_{2}=1.5, \mu_{3}=1.5$ and $\mu_{4}=1$. (S - Saddle, C - Centre)

\begin{tabular}{|c|c|c|c|c|c|c|c|c|}
\hline Point & $\tilde{q}_{1}$ & $\tilde{q}_{2}$ & $\tilde{q}_{3}$ & $\lambda_{1,2}$ & $\lambda_{3,4}$ & $\lambda_{5,6}$ & $\bar{V}$ & Type \\
\hline$\overline{E_{0}}$ & 0 & 0 & 0 & \pm 0.784 & \pm 2.210 & \pm 1.581 & 0 & $8 \times \mathrm{S} \times \mathrm{S}$ \\
\hline$E_{1}$ & 1 & 1 & 1 & \pm 2 & $\pm 0.707 \mathrm{i}$ & $\pm 1.225 \mathrm{i}$ & -0.5 & $\mathrm{~S} \times \mathrm{C} \times \mathrm{C}$ \\
\hline$E_{2}$ & -1 & -1 & -1 & \pm 2 & $\pm 0.707 \mathrm{i}$ & $\pm 1.225 \mathrm{i}$ & -0.5 & $\mathrm{~S} \times \mathrm{C} \times \mathrm{C}$ \\
\hline$E_{3}$ & 0 & $\sqrt{6} / 2$ & 0 & \pm 0.831 & $\pm 2.948 \mathrm{i}$ & $\pm 1.414 \mathrm{i}$ & -1.125 & $\mathrm{~S} \times \mathrm{C} \times \mathrm{C}$ \\
\hline$E_{4}$ & 0 & $-\sqrt{6} / 2$ & 0 & \pm 0.831 & $\pm 2.948 \mathrm{i}$ & $\pm 1.414 \mathrm{i}$ & -1.125 & $\mathrm{~S} \times \mathrm{C} \times \mathrm{C}$ \\
\hline$E_{5}$ & 1 & $\sqrt{6} / 2+1$ & 1 & $\pm 3.126 \mathrm{i}$ & $\pm 1.108 \mathrm{i}$ & $\pm 2.236 \mathrm{i}$ & -1.625 & $\mathrm{C} \times \mathrm{C} \times \mathrm{C}$ \\
\hline$E_{6}$ & 1 & $1-\sqrt{6} / 2$ & 1 & $\pm 3.126 \mathrm{i}$ & $\pm 1.108 \mathrm{i}$ & $\pm 2.236 \mathrm{i}$ & -1.625 & $\mathrm{C} \times \mathrm{C} \times \mathrm{C}$ \\
\hline$E_{7}$ & -1 & $-\sqrt{6} / 2-1$ & -1 & $\pm 3.126 \mathrm{i}$ & $\pm 1.108 \mathrm{i}$ & $\pm 2.236 \mathrm{i}$ & -1.625 & $\mathrm{C} \times \mathrm{C} \times \mathrm{C}$ \\
\hline$E_{8}$ & -1 & $\sqrt{6} / 2-1$ & -1 & $\pm 3.126 \mathrm{i}$ & $\pm 1.108 \mathrm{i}$ & $\pm 2.236 \mathrm{i}$ & -1.625 & $\mathrm{C} \times \mathrm{C} \times \mathrm{C}$ \\
\hline$E_{9}$ & $\sqrt{5} / 2$ & 0 & $-\sqrt{5} / 2$ & $\pm 2.818 \mathrm{i}$ & $\pm 1.248 \mathrm{i}$ & $\pm 2.236 \mathrm{i}$ & -1.563 & $\mathrm{C} \times \mathrm{C} \times \mathrm{C}$ \\
\hline$E_{10}$ & $-\sqrt{5} / 2$ & 0 & $\sqrt{5} / 2$ & $\pm 2.818 \mathrm{i}$ & $\pm 1.248 \mathrm{i}$ & $\pm 2.236 \mathrm{i}$ & -1.563 & $\mathrm{C} \times \mathrm{C} \times \mathrm{C}$ \\
\hline$E_{11}$ & $(\sqrt{5}+1) / 4$ & 1 & $(1-\sqrt{5}) / 4$ & $\pm 2.525 \mathrm{i}$ & $\pm 0.487 \mathrm{i}$ & \pm 1.365 & -0.844 & $\mathrm{~S} \times \mathrm{C} \times \mathrm{C}$ \\
\hline$E_{12}$ & $(1-\sqrt{5}) / 4$ & 1 & $(\sqrt{5}+1) / 4$ & $\pm 2.525 \mathrm{i}$ & $\pm 0.487 \mathrm{i}$ & \pm 1.365 & -0.844 & $\mathrm{~S} \times \mathrm{C} \times \mathrm{C}$ \\
\hline$E_{13}$ & $(\sqrt{5-1}) / 4$ & -1 & $-(\sqrt{5}+1) / 4$ & $\pm 2.525 \mathrm{i}$ & $\pm 0.487 \mathrm{i}$ & \pm 1.365 & -0.844 & $\mathrm{~S} \times \mathrm{C} \times \mathrm{C}$ \\
\hline$E_{14}$ & $-(\sqrt{5}+1) / \iota$ & -1 & $(\sqrt{5-1}) / 4$ & $\pm 2.525 \mathrm{i}$ & $\pm 0.487 \mathrm{i}$ & \pm 1.365 & -0.844 & $\mathrm{~S} \times \mathrm{C} \times \mathrm{C}$ \\
\hline$E_{15}$ & $(\sqrt{5}+1) / 4$ & -0.5 & $(1-\sqrt{5}) / 4$ & $\pm 2.707 \mathrm{i}$ & $\pm 0.376 \mathrm{i}$ & \pm 1.648 & -0.844 & $\mathrm{~S} \times \mathrm{C} \times \mathrm{C}$ \\
\hline$E_{16}$ & $(1-\sqrt{5}) / 4$ & -0.5 & $(\sqrt{5}+1) / 4$ & $\pm 2.707 \mathrm{i}$ & $\pm 0.376 \mathrm{i}$ & \pm 1.648 & -0.844 & $\mathrm{~S} \times \mathrm{C} \times \mathrm{C}$ \\
\hline$E_{17}$ & $-(\sqrt{5}+1) / \iota$ & 0.5 & $(\sqrt{5}-1) / 4$ & $\pm 2.707 \mathrm{i}$ & $\pm 0.376 \mathrm{i}$ & \pm 1.648 & -0.844 & $\mathrm{~S} \times \mathrm{C} \times \mathrm{C}$ \\
\hline$E_{18}$ & $(\sqrt{5}-1) / 4$ & 0.5 & $-(\sqrt{5}+1) / 4$ & $\pm 2.707 \mathrm{i}$ & $\pm 0.376 \mathrm{i}$ & \pm 1.648 & -0.844 & $\mathrm{~S} \times \mathrm{C} \times \mathrm{C}$ \\
\hline$E_{19}$ & $-\sqrt{14} / 7$ & $3 \sqrt{14} / 14$ & $\sqrt{14} / 7$ & $\pm 2.669 \mathrm{i}$ & \pm 0.308 & \pm 1.474 & -0.844 & $\mathrm{~S} \times \mathrm{S} \times \mathrm{C}$ \\
\hline$E_{20}$ & $\sqrt{14} / 7$ & $3 \sqrt{14} / 14$ & $-\sqrt{14} / 7$ & $\pm 2.669 \mathrm{i}$ & \pm 0.308 & \pm 1.474 & -0.844 & $\mathrm{~S} \times \mathrm{S} \times \mathrm{C}$ \\
\hline$E_{21}$ & $-\sqrt{14} / 7$ & $-3 \sqrt{14} / 14$ & $\sqrt{14} / 7$ & $\pm 2.669 \mathrm{i}$ & \pm 0.308 & \pm 1.474 & -0.844 & $\mathrm{~S} \times \mathrm{S} \times \mathrm{C}$ \\
\hline$E_{22}$ & $\sqrt{14} / 7$ & $-3 \sqrt{14} / 14$ & $-\sqrt{14} / 7$ & $\pm 2.669 \mathrm{i}$ & \pm 0.308 & \pm 1.474 & -0.844 & $\mathrm{~S} \times \mathrm{S} \times \mathrm{C}$ \\
\hline$E_{23}$ & 0.61914 & 0.88641 & 1.15367 & \pm 0.734 & \pm 1.888 & $\pm 1.450 \mathrm{i}$ & -0.482 & $\mathrm{~S} \times \mathrm{S} \times \mathrm{C}$ \\
\hline$E_{24}$ & -0.61914 & -0.88641 & -1.15367 & \pm 0.734 & \pm 1.888 & $\pm 1.450 \mathrm{i}$ & -0.482 & $\mathrm{~S} \times \mathrm{S} \times \mathrm{C}$ \\
\hline$E_{25}$ & 1.15367 & 0.88641 & 0.61914 & \pm 0.734 & \pm 1.888 & $\pm 1.450 \mathrm{i}$ & -0.482 & $\mathrm{~S} \times \mathrm{S} \times \mathrm{C}$ \\
\hline$E_{26}$ & -1.15367 & -0.88641 & -0.61914 & \pm 0.734 & \pm 1.888 & $\pm 1.450 \mathrm{i}$ & -0.482 & $\mathrm{~S} \times \mathrm{S} \times \mathrm{C}$ \\
\hline
\end{tabular}

\subsection{Numerical solution}

We first use the $4^{\text {th }}$ order polynomial to approximate a heteroclinic connection through $E_{10}$ between $E_{12}$ and $E_{13}$. We can therefore define conditions on the polynomial which approximate the heteroclinic connection, viz 


$$
\left[\begin{array}{lllll}
\boldsymbol{q}^{*}(0) & \boldsymbol{q}^{*}(T / 2) & \boldsymbol{q}^{*}(\mathrm{~T}) & \dot{\boldsymbol{q}}^{*}(0) & \dot{\boldsymbol{q}}^{*}(\mathrm{~T})
\end{array}\right]=\left[\begin{array}{ccc}
-0.309 & 1 & 0.809 \\
-1.118 & 0 & 1.118 \\
-0.809 & -1 & 0.309 \\
0 & 0 & 0 \\
0 & 0 & 0
\end{array}\right]^{\mathrm{T}}
$$

The manoeuvre duration is again set as $T=20$ and the constant gains are defined as $g_{11}=g_{21}=0.25$, $g_{12}=g_{22}=0.75$. The approximate heteroclinic connection can be seen in Fig. 18(a), where the controller tracks the approximate trajectory defined by the $4^{\text {th }}$ order polynomials. The corresponding shape of the structure during the transition from $E_{12}$ to $E_{13}$ is shown in Fig. 18(b). The labels in Fig. 18(b) present the transition process corresponding to the positions marked in Fig. 18(a), while the corresponding controls $\mu_{1}, \mu_{2}, \mu_{3}$ and $\mu_{4}$ are shown in Fig. 18(c). The corresponding mass displacements and the reference path is shown in Fig. 18(d).

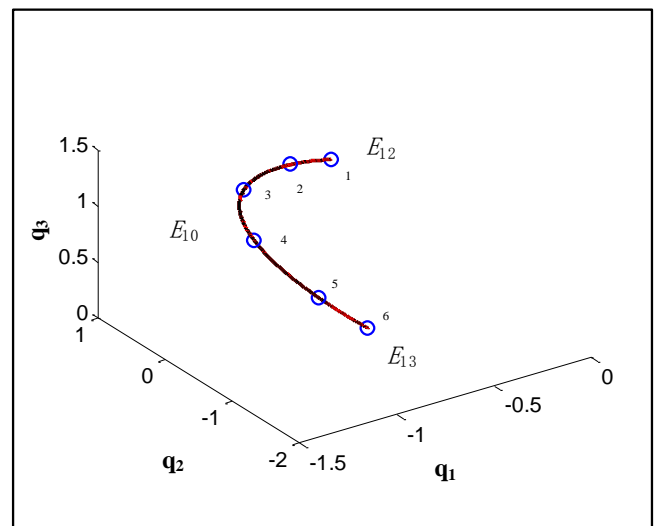

a

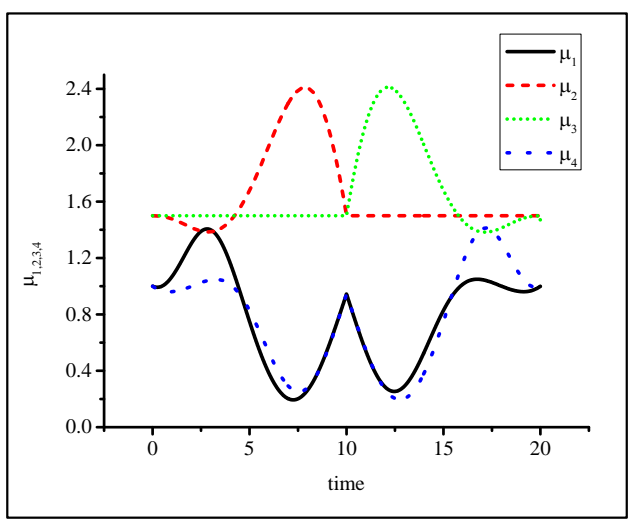

c
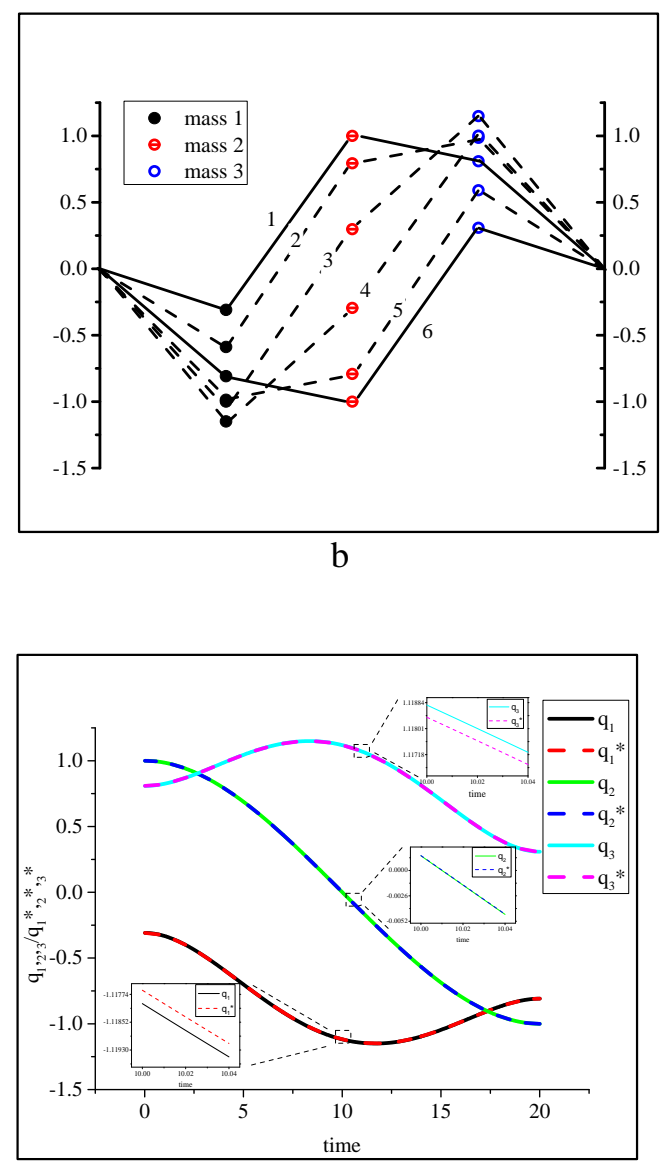

d

Figure 18. $4^{\text {th }}$ order polynomials as reference trajectory from unstable equilibrium $E_{12}$ to unstable equilibrium $E_{13}$ (a) Controlled transition, (b) Geometry of transition process, (c) Controls actuated through parameters $\mu_{1}, \mu_{2}, \mu_{3}$ and $\mu_{4}$, (d) Mass displacements during the transition from $E_{12}$ to $E_{13}$ with the reference trajectories.

Then, the method discussed in Section 3.5 is used to construct an $8^{\text {th }}$ order polynomial with the additional conditions 


$$
\left[\begin{array}{ll}
\ddot{\boldsymbol{q}}^{*}(0) & \ddot{\boldsymbol{q}}^{*}(\mathrm{~T})
\end{array}\right]=\left[\begin{array}{ll}
0 & 0 \\
0 & 0 \\
0 & 0
\end{array}\right]
$$

Moreover, using the optimisation algorithm, Fig. 19(a) shows the heteroclinic connection approximated with the $8^{\text {th }}$ order polynomial, where the gains are $g_{11}=g_{21}=0.25, g_{12}=g_{22}=0.75$. Figure 19(b) illustrates the corresponding shape of the structure during the transition from $E_{12}$ to $E_{13}$ and the corresponding controls $\mu_{1}, \mu_{2}, \mu_{3}$ and $\mu_{4}$ are shown in Fig. 19(c). It can be seen that the controls are again symmetric about $t=T / 2$ as expected. The corresponding mass displacement and the reference path is shown in Fig. 19(d).

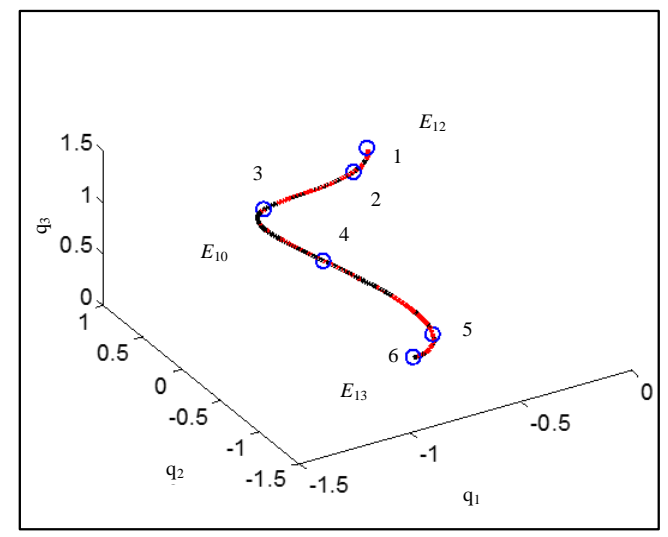

a

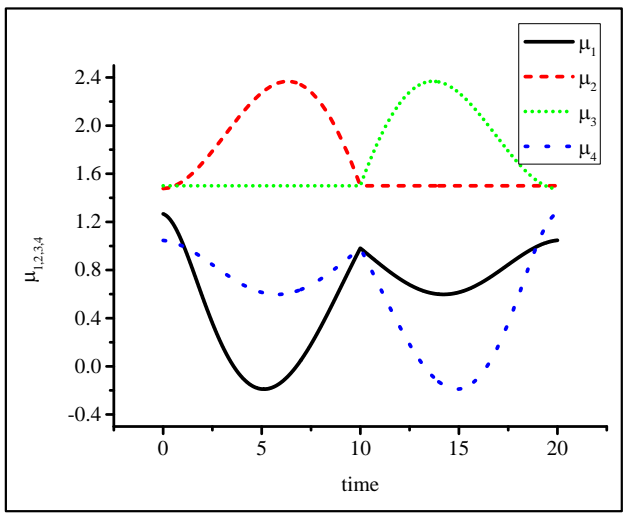

C
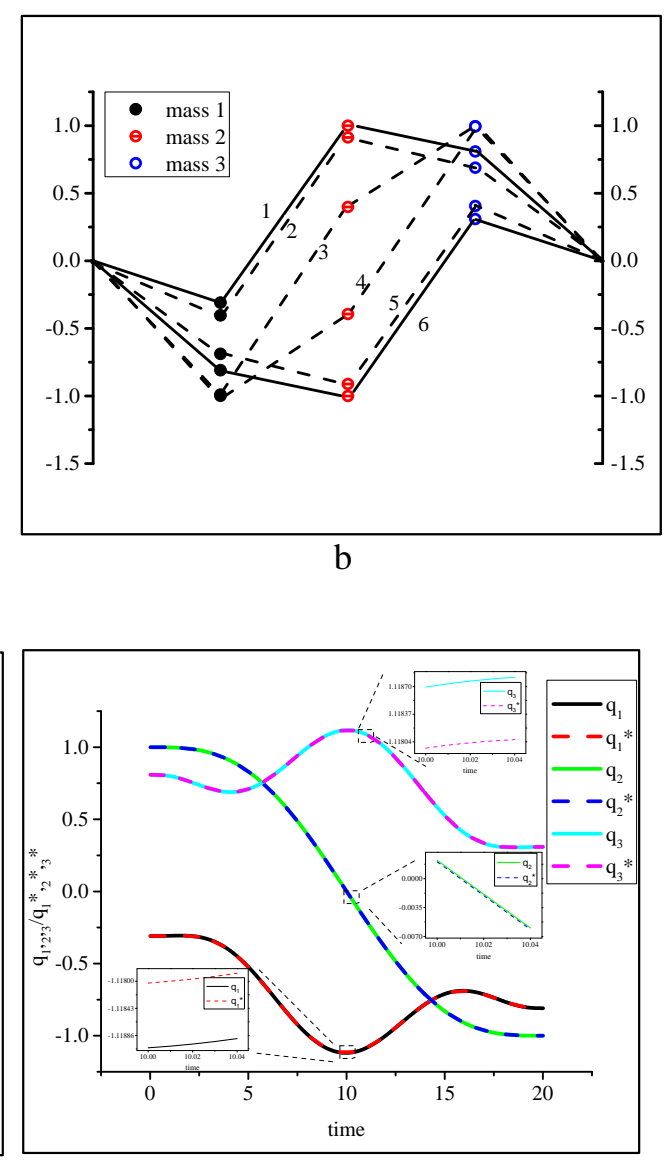

d

Figure 19. $8^{\text {th }}$ order polynomials as reference trajectory from unstable equilibrium $E_{12}$ to unstable equilibrium $E_{13}$ (a) Controlled transition, (b) Geometry of transition process, (c) Controls actuated through the coupling parameter $\mu_{1}, \mu_{2}, \mu_{3}$ and $\mu_{4}$, (d) Mass displacements during the transition from $E_{12}$ to $E_{13}$ with the reference trajectories.

The energy evaluation criteria can then be used to measure the total energy input to control the reconfiguration process, as can be seen in Fig. 20, with the $4^{\text {th }}$ order polynomials indicated as the solid line, and the $8^{\text {th }}$ order polynomials indicated as the dash line. From Fig. 20 it can be seen that a higher 
order polynomial can be used as a reference trajectory to reconfigure the 3 mass chain with significantly less energy input.

Figure 21 shows three distinct curves which define three types of different order reference trajectory with different manoeuvre durations. Again, there is an evident decrease to a minimum energy duration and then an increase as the manoeuvre duration grows similarly to the two mass problem, again as expected.

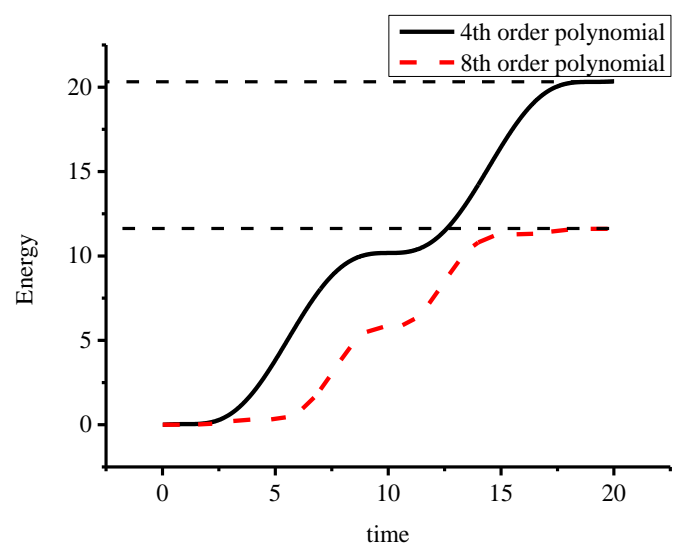

Figure 20. Comparison of energy input to track different approximate trajectories.

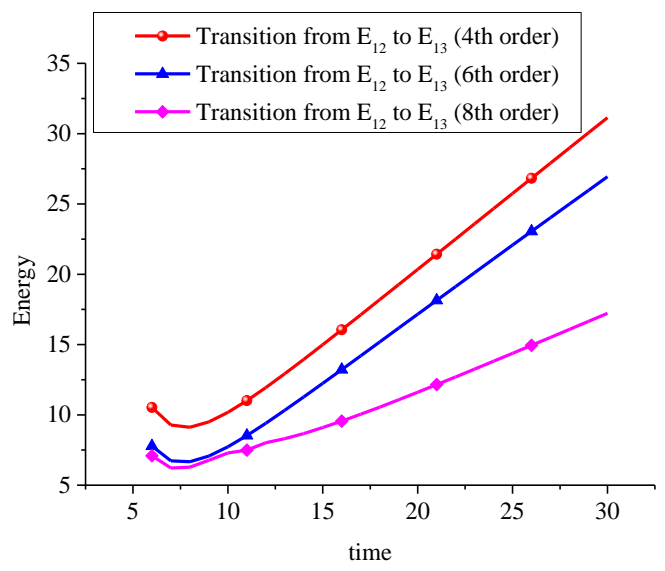

Figure 21. Comparison of energy to track different approximate trajectories.

\section{Conclusions}

A new concept for the reconfiguration of smart structures using polynomial series to approximate phase space connections has been presented. As an application for the method, a simple two mass model is firstly investigated and then a relatively complex 3 mass model used to verify that polynomial series can offer efficient reference trajectories between unstable equilibria. In addition, inverse control 
methods have been investigated to control the model for reconfiguration from one equilibrium state to another. Then an energy evaluation criteria has been employed to determine the performance of the different reference trajectories used and demonstrate that more efficient and accurate reference trajectories can be expressed by higher order polynomials. While the models used in the paper are relatively simple, they provide an approach to provide insights into low energy reconfiguration which can be extended to achieve reconfiguration of real smart structures.

\section{References}

[1] Lagoudas D C 2008 Shape Memory Alloys: Modeling and Engineering Applications (New York: Springer)

[2] Flatau A B and Chong K P 2002 Dynamic smart material and structural systems Eng. Struct. 24 261-70

[3] Qiu Z, Ling D, Zhang X and Han J 2015 Vibration control of two-connected piezoelectric flexible plate using nonlinear algorithm and T-S fuzzy controller J. Intell. Mater. Syst. Struct. 26 219-43

[4] Preumont A and Seto K 2008 Active Control of Structures (John Wiley \& Sons)

[5] Aguiar R A, Savi M A and Pacheco P M 2013 Experimental investigation of vibration reduction using shape memory alloys J. Intell. Mater. Syst. Struct. 24 247-61

[6] Lin M, Qing X, Kumar A and Beard S J 2001 SMART layer and SMART suitcase for structural health monitoring applications Proceedings of the SPIE (International Society for Optics and Photonics) pp 98-106

[7] Zhao X, Gao H, Zhang G, Ayhan B, Yan F, Kwan C and Rose J L 2007 Active health monitoring of an aircraft wing with embedded piezoelectric sensor/actuator network: I. Defect detection, localization and growth monitoring Smart Mater. Struct. 16 1208-17

[8] Donthireddy P and Chandrashekhara K 1996 Modeling and shape control of composite beams with embedded piezoelectric actuators Compos. Struct. 35 237-44

[9] Beeby S, Tudor M and White N 2006 Energy harvesting vibration sources for microsystems applications 17 175-95

[10] Hogg T and Huberman B A 1998 Controlling smart matter Smart Mater. Struct. 7 R1-14

[11] Camescasse B, Fernandes A and Pouget J 2013 Bistable buckled beam: Elastica modeling and analysis of static actuation Int. J. Solids Struct. 50 2881-93

[12] Camescasse B, Fernandes A and Pouget J 2014 Bistable buckled beam and force actuation: Experimental validations Int. J. Solids Struct. 51 1750-7

[13] Yoon H and Washington G 2010 An optimal method of shape control for deformable structures with an application to a mechanically reconfigurable reflector antenna Smart Mater. Struct. 19105004

[14] Yoon H 2013 Optimal shape control of adaptive structures for performance maximization Struct. Multidiscip. Optim. 48 571-80

[15] Kudva J N 2004 Overview of the DARPA Smart Wing Project J. Intell. Mater. Syst. Struct. 15 261-7 
[16] Hawkes E, An B, Benbernou N M, Tanaka H, Kim S, Demaine E D, Rus D and Wood R J 2010 Programmable matter by folding. Proc. Natl. Acad. Sci. U. S. A. 107 12441-5

[17] Felton S, Tolley M, Demaine E, Rus D and Wood R 2014 A method for building self-folding machines Science. $345644-6$

[18] McInnes C R and Waters T J 2008 Reconfiguring smart structures using phase space connections Smart Mater. Struct. 17025030

[19] Guenther O, Hogg T and Huberman B A 1997 Controls for unstable structures Smart Structures and Materials 1997: Mathematics and Control in Smart Structures, The Society of Photo-Optical Instrumentation Engineers (SPIE) conf. (June); Proc.SPIE pp 754-63

[20] McInnes C R, Gorman D G and Cartmell M P 2008 Enhanced vibrational energy harvesting using nonlinear stochastic resonance J. Sound Vib. 318 655-62

[21] Wiggins S 1990 Introduction to applied nonlinear dynamical systems and chaos (New York: Springer)

[22] McInnes C R 1998 Satellite attitude slew manoeuvres using inverse control Aeronaut. J. 102 259-65

[23] Aranda-Bricaire E, Moog C H and Pomet J-B 1995 A linear algebraic framework for dynamic feedback linearization IEEE Trans. Automat. Contr. 40 127-32

[24] Giurgiutiu V, Rogers C A and Chaudhry Z 1996 Energy-Based Comparison of Solid-State InducedStrain Actuators J. Intell. Mater. Syst. Struct. 7 4-14

[25] Byrd R, Hribar M and Nocedal J 1999 An interior point algorithm for large-scale nonlinear programming SIAM J. Optim. $9877-900$

[26] Murray R 2009 Optimization Based-control (California Institute of Technology)

[27] Aljonubi K, AlAmoudj A O, Langle R J and Reaney I 2013 Reconfigurable Antenna Using Smart Material 7th European Conference on Antennas and Propagation (EuCAP) pp 917-8

[28] Jakubczyk B B 1987 Feedback linearization of discrete-time systems Syst. Control Lett. 9 411-6

[29] Henk. N and Schaft A V der. 2013 Nonlinear Dynamical Control Systems (Springer Science \& Business Media)

[30] Slotine J-J E and Li W 1991 Applied Nonlinear Control (Prentice-Hall, Inc.) 